\title{
Una nueva versión de la escala Percepción del Alumnado sobre el Clima Escolar (PACE) y análisis de sus propiedades psicométricas Naiara Escalante-Mateos*, Arantza Fernández-Zabala, Eider Goñi-Palacios e
Iker Izar de la Fuente-Díaz de Cerio \\ Facultad de Educación y Deporte - Universidad del País Vasco (UPV/EHU).
}

Resumen: El clima escolar es un factor fundamental para la calidad, eficacia y mejora del centro escolar. Por ello, muchos estudios subrayan la importancia de evaluar este constructo adecuadamente. Sin embargo, se comprueba que aún no se cuenta con un instrumento válido y fiable en castellano que refleje por completo los aspectos principales del clima escolar que cuentan con una mayor aprobación entre la comunidad científica. Para tratar de superar esta limitación, los objetivos principales del presente estudio son rediseñar la escala Percepción del Alumnado sobre el Clima Escolar (PACE) y presentar evidencias de su fiabilidad y validez en población adolescente española. Participaron 348 estudiantes de 12 a 18 años, que cumplimentaron dos autoinformes: la nueva versión de la escala PACE y el Cuestionario para Evaluar el Clima Social del Centro Escolar (CECSCE). Los resultados obtenidos permiten seleccionar los ítems con mejores garantías técnicas para la evaluación de las nueve subescalas obtenidas. En consecuencia, se obtiene una escala válida, fiable y breve que evalúa la percepción del alumnado sobre las dimensiones específicas de los aspectos mayormente aceptados del clima escolar. Esta escala supone además un aporte importante en la clarificación de la estructura interna del clima escolar.

Palabras clave: Clima escolar, Auto-informe, Confiabilidad, Validez, Análisis factorial exploratorio.

\section{A new version of the Students' Perception of School Climate scale (PACE) and an analysis of its psychometric properties}

\begin{abstract}
The school climate is a fundamental factor for the quality, efficiency and improvement of the school. Hence, many studies stress the importance of evaluating this construct properly. However, there is still no valid and reliable instrument in Spanish that fully reflects the main aspects of school climate and that is approved by most of the scientific community. To try to overcome this limitation, the main objectives of this study are to redesign the Students' Perception of School Climate (PACE) scale and to present evidence of its reliability and validity in the Spanish adolescent population. A total of 348 students aged 12 and 18 years participated in the study and completed two self-reports: the new version of the PACE scale and the School Climate Assessment Questionnaire (CECSCE). The results obtained allowed us to select the items with the best technical guarantees for the evaluation of the nine subscales obtained. Consequently, a valid, reliable and brief scale is obtained that evaluates the students' perception of the specific dimensions of the most highly accepted aspects of the school climate. This scale also makes an important contribution to clarifying the internal structure of the school climate.

Keywords: School climate, Self-report, Reliability, Validity, Exploratory factor analysis.
\end{abstract}

Está ampliamente documentado que son múltiples los resultados beneficiosos para el alumnado que conlleva un clima escolar

Recibido: 10/03/2020 - Aceptado: 26/05/2020 - Avance online: 15/06/2020 *Correspondencia: Naiara Escalante Mateos

Facultad de Educación y Deporte - Universidad del País Vasco (UPV/EHU)

C.P: 01006, Vitoria-Gasteiz, España.

E-mail: naiara.escalante@ehu.eus

Escalante-Mateos, N., Fernández-Zabala, A., Goñi-Palacios, E. e lzar de la Fuente-Díaz de Cerio, I. (2020). Una nueva versión de la escala Percepción del Alumnado sobre el Clima Escolar (PACE) y análisis de sus propiedades psicométricas. Revista de Psicología y Educación 15(2), 201-224, https://doi.org/10.23923/rpye2020.02.196 positivo (Maxwell, Reynolds, Lee, Subasic, y Bromhead, 2017; Sun y Royal, 2017; Thapa, Cohen, Guffey, y Higgins-D'Alessandro, 2013). Se ha demostrado que el clima escolar es un factor protector frente a la victimización y el acoso escolar (Farina, 2019; Hong, Espelage, y Lee, 2018) y se ha asociado, entre otras muchas variables, con un mejor bienestar

1699-9517/@ 2020 Asociación Científica de Psicología y Educación (ACIPE). Publicado por Consejo General de Colegios Oficiales de Psicólogos, España. Este es un artículo Open Access bajo la CC BY-NC-ND licencia (http://creativecommons.org/licencias/bync-nd/4.0/ 
psicológico del alumnado (Newland, DeCino, Mourlam, y Strous, 2019; Varela et al.,2019), con mejores logros académicos (Daily, Mann, Kristjansson, Smith, y Zullig, 2019; López-González y Oriol, 2016) y una mayor adaptación escolar y conductas prosociales (Herrera y Rico, 2014; Turner, Reynolds, Lee, Subasic, y Bromhead, 2014 ). Por ello, diversos autores subrayan la importancia de medir con precisión este constructo con el objetivo de mejorar la calidad de los centros educativos (Sun y Royal, 2017). Sin embargo, este quehacer resulta especialmente difícil debido fundamentalmente a que el clima escolar es un constructo multidimensional que ha sido examinado desde diferentes perspectivas teóricas y metodológicas (Maxwell et al., 2017).

El clima escolar ha sido definido e incluso denominado de maneras muy dispares a lo largo de los años (clima de convivencia, atmosfera escolar, ambiente escolar, etc.), por lo que existe una gran cantidad de definiciones de este constructo (Cohen, McCabe, Michelli, y Pickeral, 2009; Sun y Royal, 2017). No obstante, aunque no existe una definición ampliamente aceptada, algunos de los referentes más importantes en el estudio del clima escolar lo definen como un constructo multidimensional que se basa en la percepción que poseen los individuos acerca de su centro escolar a partir de las experiencias vividas en él y que refleja los valores, objetivos, normas, costumbres, relaciones interpersonales, prácticas de enseñanza y aprendizaje y estructuras organizativas del centro escolar (Cohen et al., 2009; National School Climate Center, 2014).

La falta de una definición única y unívoca del clima escolar ha derivado en que hoy en día aún no se conozca con exactitud la estructura interna de este constructo, y por tanto, en que se mida de manera inconsistente (Maxwell et al., 2017; Thapa et al., 2013). Existe una gran cantidad y heterogeneidad de instrumentos de medida del clima escolar, puesto que, desde la década de los 80 , se han diseñado multitud de escalas con sus correspondientes subescalas que fluyen desde diferentes articulaciones del constructo
(Maxwell et al., 2017). Sin embargo, la mayoría de los estudios coinciden en afirmar que el clima escolar está constituido por cuatro aspectos generales de la vida escolar: seguridad, relaciones, aspectos ambientalesestructurales y enseñanza-aprendizaje. Se admite, además, que esos cuatro aspectos se componen de dimensiones más específicas (Cohen, 2006; Cohen et al., 2009; National School Climate Center, 2014), siendo las siguientes algunos ejemplos de las mismas: relación profesorado-alumnado (LópezGonzález y Bisquerra, 2013; Quijada, Ruiz, Huertas, Alonso, y Tapia, 2020); relación entre iguales (Yang et al., 2013, Quijada et al, 2020); cohesión ente iguales/ cooperación (López-González y Bisquerra, 2013; LópezGonzález, Tourón y Tejedor, 2012); equidad de las reglas (Yang et al., 2013; Bear, Gaskins, Blank, y Chen, 2011); disciplina (Aldridge y Ala'i, 2013); seguridad (Bear et al., 2011; Guo, Choe, y Higgins-D'Alessandro, 2011); ambiente físico (López-González et al., 2012); expectativas del profesorado (Bear et al., 2011; López-González et al., 2012); competitividad (López-González et al., 2012; Pérez, Ramos, y López, 2010); motivación del logro (Haynes, Emmons, y Comer, 1993) o recursos (Cemalcilar, 2010; Haynes et al., 1993).

Esta propuesta conceptual tan variada ha derivado en la creación de numerosos y muy dispares instrumentos de medida para la evaluación de la percepción del alumnado sobre el clima escolar. No obstante, tras la revisión de diversas medidas diseñadas en distintos países e idiomas, se concluye que hoy en día, aún, no se conoce con exactitud la estructura interna del clima escolar y que además, no se dispone de ningún instrumento válido y fiable en castellano que evalúe de manera equilibrada las dimensiones específicas de los cuatro aspectos principales que la mayoría de las investigaciones defienden como componentes del clima escolar. Se observa que la mayor parte de las medidas proceden de Estados Unidos y que son escasas las que han sido validadas en el contexto español (e.g., ECLIS: Aron, Milicic, y Armijo, 2012; EBCC: López-González y 
Bisquerra, 2013). Además, se comprueba que la mayor parte de éstas determinan el clima escolar fundamentalmente desde la percepción que posee el alumnado sobre la aplicación consistente de normas y sobre las relaciones que mantiene con sus iguales y con el profesorado, sin reparar en otros muchos aspectos que son esenciales para la creación y permanencia de un clima escolar positivo (Escalante, Fernández-Zabala, Goñi, e Izarde-la-Fuente, 2020).

Para solventar las limitaciones detectadas, se diseñó un nuevo instrumento de medida, la escala Percepción del Alumnado sobre el Clima Escolar (PACE), y se analizaron sus propiedades psicométricas en un estudio piloto (Escalante, Goñi, Fernández-Zabala, e Izar-de-la-Fuente, 2020). Dado que en dicho estudio esta escala mostró características susceptibles de ser mejoradas, el objetivo de este trabajo es doble: rediseñar la escala PACE y presentar evidencias de su fiabilidad y validez en población adolescente española.

\section{MÉTODO}

\section{DISEÑO}

Este trabajo se corresponde con un estudio instrumental, puesto que está encaminado al diseño y al análisis de las propiedades psicométricas de una escala de medida (Montero y León, 2007).

\section{PARTICIPANTES}

Inicialmente participaron un total de 365 estudiantes de Educación Secundaria Obligatoria (ESO) y Bachillerato pertenecientes a siete institutos: seis de la Comunidad Autónoma del País Vasco, tres públicos y tres concertados; y uno público del Principado de Asturias. No obstante, debido a la eliminación de los sujetos que no habían respondido a más del 10\% de los ítems y de los outliers (sujetos con respuestas muy extremas o inconsistentes), la muestra finalmente quedó compuesta por 348 estudiantes con edades comprendidas entre los 12 y los 18 años $(M=15.05$ años, $D T=1.97)$. El $55.8 \%$ de las personas participantes eran mujeres y el $44.2 \%$ hombres, considerándose grupos equilibrados $\left(\chi^{2}=4.17, p>.05\right)$. De esta muestra, 160 estudiantes contestaron al cuestionario pensado para someter a prueba la validez convergente de la nueva versión de la escala PACE. La selección de la muestra se realizó de forma incidental.

\section{INSTRUMENTOS DE MEDIDA}

El clima escolar fue evaluado con la nueva versión de la escala PACE (Anexo 1). Este instrumento consta de nueve subescalas, compuestas por 68 ítems (siete inversos y 61 directos): (1) Seguridad física; (2) Normas; (3) Relación alumnado-profesorado; (4) Relación entre iguales; (5) Cohesión de grupo; (6) Aspectos ambientales-estructurales; (7) Capacidad de motivación del profesorado; (8) Expectativas del profesorado; y (9) Recursos metodológicos. Cuenta con un formato de respuesta en escala Likert de cinco grados donde 1 = Totalmente en desacuerdo; 2 = En desacuerdo; $3=\mathrm{Ni}$ de acuerdo, ni desacuerdo; $4=$ De acuerdo; y $5=$ Totalmente de acuerdo.

Para analizar la validez convergente de la nueva versión de la escala PACE, se utilizó el Cuestionario para Evaluar el Clima Social del Centro Escolar (CECSCE) de Trianes, Blanca, de la Morena, Infante, y Raya (2006) ya que, a pesar de que ha sido validado por sus autoras únicamente en $2^{\circ}$ y $3^{\circ}$ de la ESO y evalúa sólo de manera muy general los aspectos que la mayoría de las personas investigadoras afirman que componen el clima escolar, es uno de los cuestionarios más utilizados en la evaluación de este constructo. El cuestionario se compone de 14 ítems extraídos del California School Climate and Safety Survey (CSCSS; Furlong y Morrison, 1995; Rosenblatt y Furlong, 1997). Esos ítems son medidos en una escala Likert de cinco grados que oscila desde $1=$ Nunca a $5=$ Muchas veces. Distingue dos factores con adecuados índices de consistencia interna, los siguientes en el caso de la muestra del presente trabajo: clima referente al centro $(\alpha=, 796)$ y clima referente al profesorado $(\alpha=, 784)$. 


\section{PROCEDIMIENTO}

Este estudio se llevó a cabo en dos fases diferenciadas. La primera, consistió en el rediseño de la escala PACE. La segunda, residió en el análisis de las propiedades psicométricas de la nueva versión de la escala en una muestra de estudiantes de ESO y Bachillerato. Para poder llevar a cabo este cometido, dado que era precisa la participación de menores de edad, el primer paso consistió en obtener el informe favorable del Comité de Ética para las Investigaciones relacionadas con Seres Humanos de la Universidad del País Vasco (CEISH-UPV/EHU). Una vez conseguido dicho permiso, el personal investigador de este estudio se puso en contacto con distintos institutos, tanto públicos como concertados, para pedir su colaboración en la investigación. A continuación, a los centros interesados se les pidió el consentimiento oportuno para administrar entre su alumnado los instrumentos de medida. Se solicitó también el permiso de los progenitores o tutores legales y el del propio alumnado participante. Seguidamente, dos de las investigadoras de este estudio se desplazaron a los centros que accedieron a participar en la investigación y procedieron a la administración de los instrumentos de medida en horario lectivo, de manera simultánea a todo el alumnado integrante de una misma aula, con el fin de asegurar la uniformidad en la aplicación. El tiempo de respuesta a los instrumentos osciló entre 15 y 20 minutos. Durante la recolección de los datos se siguió el criterio de ciego único y se garantizó el carácter anónimo y voluntario de la participación del alumnado, con el objetivo de disminuir la tendencia de deseabilidad social en sus respuestas.

\section{ANÁLISIS DE DATOS}

Todos los análisis realizados en este estudio se llevaron a cabo con el paquete estadístico SPSS 25 para Windows.

En primer lugar, se eliminaron aquellos sujetos que no habían respondido a más del $10 \%$ del total de ítems junto con los que mostraron patrones de respuesta extraños o inconsistentes. Esos sujetos fueron identificados mediante la prueba del análisis de clusters o conglomerados. En segundo lugar, se reemplazaron los valores perdidos (menos del 10\% del total), a través del método de estimación de tendencia lineal en el punto.

Posteriormente, para la selección de los ítems con mejores garantías técnicas, se siguieron distintos cometidos. En primer lugar, se realizó un análisis descriptivo de los ítems para conocer si cada ítem que conforma la nueva versión de la escala PACE tiene capacidad discriminativa y representa todos los niveles en la autopercepción concreta a la que se refiere, es decir de 1 a 5, lo cual implica tener una media centrada (Nunnally y Bernstein, 1995), una desviación típica superior a 1 (Carretero-Dios y Pérez, 2005), una asimetría no superior ni muy cercana al valor absoluto de 2 (Bandalos y Finney, 2010; Gravetter y Wallnau, 2014) y una curtosis entre -5 y 5 (Bentler, 2005).

En segundo lugar, se examinó la correlación de cada ítem con su propio factor (discriminación) y con el resto de factores (homogeneidad). Para considerarse adecuadas, las correlaciones referentes a la discriminación deben obtener valores superiores a .25 - . 30 (Nunnally y Bernstein, 1995) y las correspondientes a la homogeneidad deben presentar diferencias positivas a favor del factor propio de al menos .20 (Jackson, 1970).

En tercerlugar, se estudió la dimensionalidad de la escala. Para medir la adecuación muestral y evaluar la aplicabilidad del análisis factorial exploratorio (AFE), se utilizaron dos estimadores: KMO y Barlett. Kaiser (1970) aconseja el siguiente baremo para interpretar el índice KMO: $1 \geq \mathrm{KMO} \geq .90$, muy bueno; $.90 \geq \mathrm{KMO} \geq .80$, meritorio; $.80 \geq$ $\mathrm{KMO} \geq .70$, mediano; $.70 \geq \mathrm{KMO} \geq .60$, mediocre; $60 \geq \mathrm{KMO}>.50$, bajo; $\mathrm{KMO} \leq$ .50 , inaceptable. En la prueba de esfericidad de Bartlett, si no se acepta la hipótesis nula $(p<.05)$ significa que las variables están intercorrelacionadas y que, por tanto, es pertinente proceder al AFE (Montoya, 2007). Para la realización de este análisis, se optó por el método de estimación mínimos cuadrados 
no ponderados, el método de extracción más recomendado cuando no se cumple el supuesto de normalidad y el número de ítems es elevado (Flora, LaBrish, y Chalmers, 2012; Lloret-Segura, Ferreres-Traver, HernándezBaeza, y Tomás-Marco, 2014), como ocurre en el caso que nos ocupa. Se eligió la rotación oblicua (oblimin directo), partiendo del supuesto de que los factores de este estudio están relacionados (Lloret-Segura et al., 2014). Igualmente, se comprobó si la comunalidad de los ítems superaba el .30 exigido (Hair, Anderson, Tatham, y Black, 2004); si los ítems discriminaban adecuadamente, esto es, si sus pesos factoriales superaban el mínimo de 40 (Bandalos y Finney, 2010; Morales, 2011); y si los ítems quedaban agrupados de manera conjunta en los factores en los que se había previsto que lo hicieran.

Cabe señalar que en esta investigación se hizo uso de la matriz de correlaciones productomomento de Pearson ya que, a pesar de que los ítems no son variables continuas, cumplen con los criterios para emplear este tipo de matriz dado que presentan cinco alternativas de respuesta y distribuciones aproximadamente normales (Lloret-Segura et al., 2014).

Una vez realizada la selección de ítems, se analizó la fiabilidad de la consistencia interna de cada una de las subescalas a través del alfa de Cronbach y los índices de fiabilidad compuesta omega de McDonald y rho de Raykov, siguiendo el criterio de Cho (2016). Asimismo, se calculó la Varianza Media Extraída
(VME) como indicador de la validez de la estructura interna de la escala. Está estipulado que los distintos coeficientes de fiabilidad de consistencia interna deben presentar valores superiores a .70 para ser adecuados (Hair, Babin, Anderson y Black, 2018). En lo que respecta a la Varianza Media Extractada (VME), se consideran adecuados los valores próximos al punto de corte establecido de .50 (Hair et al., 2018).

Por último, para comprobar la validez convergente, se analizaron las relaciones bivariadas entre las subescalas de la nueva versión del PACE y las del CECSCE. El grado de asociación entre las diferentes subescalas se estableció siguiendo la clasificación ofrecida por Bisquerra (2004): <.20 muy baja; de .20 a .39 baja; de .40 a .59 moderada; de .60 a .79 alta; $y>.80$ muy alta.

\section{RESULTADOS}

\section{PRIMERA FASE. REDISEÑO DE LA ESCALA PACE}

En el rediseño de la escala PACE se siguen diferentes pasos. En primer lugar, se revisan los cinco ítems que, entre los 42 de los que queda compuesta la escala en el estudio piloto realizado (Escalante, Goñi et al., 2020), presentan propiedades mejorables. Tras analizar el contenido y la redacción de los mismos, se opta por modificar ligeramente su redacción (Tabla 1).

Tabla 1

Ítems cuya redacción ha sido modificada en la nueva versión de la escala PACE respecto a la escala original

\begin{tabular}{|c|c|}
\hline $\begin{array}{c}\text { En este centro los/las alumnos/as agreden o amenazan al } \\
\text { profesorado. }\end{array}$ & $\begin{array}{c}\text { En este centro hay alumnos/as que agreden o amenazan al } \\
\text { profesorado. }\end{array}$ \\
\hline $\begin{array}{c}\text { Tengo compañeros/as en los que puedo confiar. } \\
\text { En esta clase los/las alumnos/as tenemos buena comunicación } \\
\text { entre nosotros/as. }\end{array}$ & $\begin{array}{c}\text { Los/las alumnos/as tenemos buena comunicación entre } \\
\text { nosotros/as. }\end{array}$ \\
\hline $\begin{array}{c}\text { Los/las profesores/as creen que todos/as los/las alumnos/as } \\
\text { podemos tener éxito en el centro. }\end{array}$ & $\begin{array}{c}\text { Los/las profesores/as confían en que todos/as los/las alumnos/as } \\
\text { podemos sacar buenas notas. }\end{array}$ \\
\hline $\begin{array}{c}\text { Los/las profesores/as esperan que continuemos con nuestros } \\
\text { estudios. }\end{array}$ & \begin{tabular}{c} 
Los/las profesores/as esperan que sigamos estudiando. \\
\hline
\end{tabular}
\end{tabular}


A continuación, se elabora una lista con nuevos ítems para completar las nueve subescalas obtenidas en el estudio piloto (Tabla 2), evitando la infra o sobrerrepresentación de ninguna de estas. Se decide incorporar los ítems necesarios para que todas consten de nueve; así, se elabora un total de 39 ítems para incorporar a los 42 previamente existentes.

Seguidamente, siguiendo la recomendación de Muñiz (2018), se pide a un grupo de expertas (seis doctoras de la Universidad del País Vasco con amplio recorrido como docentes universitarias y con experiencia como educadoras, profesoras, psicólogas y orientadoras en Educación Infantil, Primaria y Secundaria, en gabinetes psicopedagógicos, etc.) que realicen una evaluación de los 81 ítems, basada en los siguientes criterios: representatividad, relevancia, diversidad, claridad, sencillez y comprensibilidad. Durante la valoración por parte de las expertas, algunos de los ítems fueron descartados, otros reformulados y otros nuevos fueron propuestos, obteniendo así una nueva versión de la escala PACE compuesta por 68 ítems, que es la que se administra en el presente estudio.

Una vez aplicada la nueva versión de la escala a la muestra de interés, se procede al análisis de las propiedades psicométricas de la misma, tal y como se expone a continuación.

\section{SEGUNDA FASE. ANÁLISIS DE LAS PROPIEDADES PSICOMÉTRICAS DE LA NUEVA VERSIÓN DE LA ESCALA PACE}

\section{CAPACIDAD DISCRIMINATIVA DE LOS ÍTEMS DE LA NUEVA VERSIÓN DE LA ESCALA PACE}

En primer lugar, se presentan diversos datos sobre las características psicométricas de los ítems que conforman la escala rediseñada del PACE (Tabla 3 ).

Las puntuaciones medias de los ítems se sitúan entre el 2.94 (PACE25) y el 4.42 (PACE52), siendo la media aritmética superior

Tabla 2

Subescalas de la escala PACE

\begin{tabular}{|c|c|}
\hline Subescala & Definición \\
\hline Seguridad física & $\begin{array}{l}\text { La percepción de peligros, daños y/o riesgos en el centro que lleva al individuo a una } \\
\text { sensación de inseguridad en la escuela. }\end{array}$ \\
\hline Normas & $\begin{array}{l}\text { Percepción que tiene cada individuo de las reglas de su centro escolar (existencia de } \\
\text { normas, comunicación de las mismas, etc.). }\end{array}$ \\
\hline Relación alumnado-profesorado & $\begin{array}{l}\text { Impresión que tiene cada alumno/a de la relación que mantiene con su profesorado } \\
\text { (comunicación, confianza, etc.). }\end{array}$ \\
\hline Relación entre iguales & $\begin{array}{l}\text { Idea que tiene cada alumno/a de la relación que mantiene con sus iguales } \\
\text { (comunicación, confianza, etc.). }\end{array}$ \\
\hline Cohesión de grupo & $\begin{array}{l}\text { Percepción que posee cada individuo de la tendencia del grupo clase a respetarse, } \\
\text { a ayudarse, y a mantenerse y permanecer unido. }\end{array}$ \\
\hline Aspectos ambientales-estructurales & $\begin{array}{l}\text { Idea que tiene cada individuo de diferentes aspectos ambientales-estructurales de } \\
\text { su centro escolar que repercuten en su bienestar y salud (limpieza de los espacios del } \\
\text { centro, iluminación, etc.). }\end{array}$ \\
\hline $\begin{array}{l}\text { Capacidad de motivación del } \\
\text { profesorado }\end{array}$ & $\begin{array}{c}\text { Percepción que posee cada individuo de las habilidades que tiene el profesorado } \\
\text { para influir en su forma de actuar (motivar, etc.), haciendo que trabaje con } \\
\text { entusiasmo hacia el logro de sus metas y objetivos. }\end{array}$ \\
\hline Percepción expectativas del profesorado & $\begin{array}{l}\text { Idea que tiene cada individuo de la esperanza pedagógica del profesorado acerca } \\
\text { del trabajo, interés por los estudios, etc. de todo el alumnado. }\end{array}$ \\
\hline Recursos metodológicos & $\begin{array}{c}\text { Percepción que posee cada alumno/a sobre los recursos metodológicos utilizados } \\
\text { por el profesorado (recursos innovadores, etc.). }\end{array}$ \\
\hline
\end{tabular}


al punto medio de la escala recomendado. En lo que respecta a la desviación típica, la mayoría de los valores superan el valor recomendado de 1. Asimismo, los coeficientes de asimetría y curtosis de todos los ítems cumplen con los criterios establecidos. Por otra parte, la eliminación de únicamente cinco ítems (PACE07, PACE 12, PACE47, PACE50, y PACE67) supondría una mejora en el valor del alfa de Cronbach general de las subescalas a las que pertenecen, lo que es un buen indicativo de la consistencia interna del instrumento.

\section{CORRELACIONES RELATIVAS A LA DISCRIMINACIÓN Y HOMOGENEIDAD DE LOS ÍTEMS DE LA NUEVA VERSIÓN DE LA ESCALA PACE}

Respecto a la discriminación y homogeneidad de los ítems, tal y como puede comprobarse en la Tabla 4, las correlaciones relativas a la discriminación obtienen valores superiores a $.25-.30$ en todos los casos y las relativas a la homogeneidad, exceptuando en 14 ítems (PACE04, PACE07, PACE13, PACE2 1, PACE23, PACE24, PACE32, PACE34, PACE4 1, PACE50, PACE51, PACE57, PACE60, y PACE65), muestran diferencias positivas a favor del factor propio de al menos .20.

Tabla 3

Características descriptivas de los ítems de la nueva versión del PACE

\begin{tabular}{|c|c|c|c|c|c|c|c|c|}
\hline & Mín & Máx & $M$ & $D T$ & Asimetría & Curtosis & $\begin{array}{c}\text { Alfa si se } \\
\text { elimina ítem }\end{array}$ & $\begin{array}{c}\text { Alfa } \\
\text { subescala }\end{array}$ \\
\hline PACEO1_Seg & 1 & 5 & 3.04 & 1.105 & -0.149 & -0.549 & .745 & \\
\hline PACE17_Seg & 1 & 5 & 3.00 & 1.026 & -0.208 & -0.504 & .726 & \\
\hline PACE25_Seg & 1 & 5 & 2.94 & 1.204 & 0.013 & -0.849 & .718 & \\
\hline PACE35_Seg & 1 & 5 & 3.19 & 1.140 & -0.277 & -0.577 & .747 & \\
\hline PACE47_Seg & 1 & 5 & 4.00 & 1.084 & -1.018 & 0.350 & .781 & \\
\hline PACE54_Seg & 1 & 5 & 3.34 & 1.089 & -0.378 & -0.394 & .720 & \\
\hline PACE67_Seg & 1 & 5 & 3.43 & 1.344 & -0.413 & -0.992 & .798 & \\
\hline Seguridad & & & & & & & & .777 \\
\hline PACEO3_Nor & 1 & 5 & 4.15 & 0.964 & -1.198 & 1.232 & .832 & \\
\hline PACE10_Nor & 1 & 5 & 4.10 & 1.106 & -1.258 & 0.962 & .834 & \\
\hline PACE20_Nor & 1 & 5 & 4.06 & 0.896 & -0.741 & 0.046 & .825 & \\
\hline PACE30_Nor & 1 & 5 & 4.09 & 1.065 & -1.153 & 0.733 & .813 & \\
\hline PACE38_Nor & 1 & 5 & 3.97 & 1.140 & -0.925 & 0.028 & .830 & \\
\hline PACE45_Nor & 1 & 5 & 4.11 & 1.007 & -1.106 & 0.866 & .837 & \\
\hline PACE52_Nor & 1 & 5 & 4.42 & 0.931 & -1.943 & 3.699 & .839 & \\
\hline PACE64_Nor & 1 & 5 & 4.27 & 0.840 & -1.138 & 1.101 & .840 & \\
\hline \multicolumn{8}{|l|}{ Normas } & .850 \\
\hline
\end{tabular}


Tabla 3 (Continuación)

Características descriptivas de los ítems de la nueva versión del PACE

\begin{tabular}{|c|c|c|c|c|c|c|c|c|}
\hline & Mín & Máx & $M$ & $D T$ & Asimetría & Curtosis & $\begin{array}{c}\text { Alfa si se } \\
\text { elimina } \\
\text { ítem }\end{array}$ & $\begin{array}{c}\text { Alfa } \\
\text { subescala }\end{array}$ \\
\hline PACEO2_Alpro & 1 & 5 & 3.32 & 1.274 & -0.309 & -0.874 & .908 & \\
\hline PACE19_Alpro & 1 & 5 & 3.54 & 1.068 & -0.429 & -0.303 & .907 & \\
\hline PACE26_Alpro & 1 & 5 & 3.06 & 1.286 & -0.179 & -0.975 & .905 & \\
\hline PACE37_Alpro & 1 & 5 & 3.35 & 1.116 & -0.323 & -0.515 & .908 & \\
\hline PACE46_Alpro & 1 & 5 & 2.99 & 1.259 & -0.056 & -0.931 & .903 & \\
\hline PACE51_Alpro & 1 & 5 & 3.46 & 1.001 & -0.444 & -0.157 & .910 & \\
\hline PACE55_Alpro & 1 & 5 & 3.54 & 1.241 & -0.542 & -0.690 & .904 & \\
\hline PACE65_Alpro & 1 & 5 & 3.24 & 1.074 & -0.140 & -0.614 & .908 & \\
\hline Alpro & & & & & & & & .917 \\
\hline PACEO5_lgu & 1 & 5 & 4.40 & 0.847 & -1.536 & 2.220 & .906 & \\
\hline PACE11_lgu & 1 & 5 & 4.00 & 1.056 & -0.992 & 0.466 & .898 & \\
\hline PACE16_lgu & 1 & 5 & 4.13 & 1.028 & -1.188 & 0.978 & .898 & \\
\hline PACE24_lgu & 1 & 5 & 4.29 & 0.938 & -1.403 & 1.761 & .908 & \\
\hline PACE28_Igu & 1 & 5 & 4.04 & 0.899 & -0.827 & 0.484 & .900 & \\
\hline PACE40_lgu & 1 & 5 & 3.90 & 1.070 & -0.816 & 0.075 & .899 & \\
\hline PACE49_Igu & 1 & 5 & 4.03 & 0.954 & -0.901 & 0.410 & .908 & \\
\hline Iguales & & & & & & & & .915 \\
\hline PACEO4_Coh & 1 & 5 & 4.06 & 0.850 & -0.531 & -0.489 & .894 & \\
\hline PACE12_Coh & 1 & 5 & 3.79 & 1.023 & -0.625 & -0.058 & .902 & \\
\hline PACE18_Coh & 1 & 5 & 3.80 & 1.008 & -0.668 & 0.089 & .885 & \\
\hline PACE27_Coh & 1 & 5 & 3.64 & 0.966 & -0.723 & 0.604 & .882 & \\
\hline PACE36_Coh & 1 & 5 & 4.08 & 0.874 & -0.939 & 1.035 & .884 & \\
\hline PACE43_Coh & 1 & 5 & 3.53 & 0.981 & -0.365 & -0.130 & .886 & \\
\hline PACE56_Coh & 1 & 5 & 3.99 & 0.955 & -0.839 & 0.388 & .886 & \\
\hline PACE62_Coh & 1 & 5 & 3.84 & 0.934 & -0.658 & 0.226 & .884 & \\
\hline \multicolumn{8}{|l|}{ Cohesión } & .901 \\
\hline
\end{tabular}


Tabla 3 (Continuación)

Características descriptivas de los ítems de la nueva versión del PACE

\begin{tabular}{|c|c|c|c|c|c|c|c|c|}
\hline & Mín & Máx & $M$ & $D T$ & Asimetría & Curtosis & \begin{tabular}{c|} 
Alfa si se \\
elimina ítem
\end{tabular} & $\begin{array}{c}\text { Alfa } \\
\text { subescala }\end{array}$ \\
\hline PACEO9_Amb & 1 & 5 & 3.58 & 1.145 & -0.444 & -0.618 & .793 & \\
\hline PACE22_Amb & 1 & 5 & 3.07 & 1.135 & -0.194 & -0.641 & .807 & \\
\hline PACE31_Amb & 1 & 5 & 3.52 & 0.970 & -0.245 & -0.313 & .795 & \\
\hline PACE39_Amb & 1 & 5 & 4.07 & 0.972 & -1.047 & 0.854 & .799 & \\
\hline PACE48_Amb & 1 & 5 & 4.06 & 0.837 & -0.648 & 0.314 & .801 & \\
\hline PACE53_Amb & 1 & 5 & 3.27 & 1.212 & -0.171 & -0.882 & .810 & \\
\hline PACE59_Amb & 1 & 5 & 3.16 & 1.230 & -0.198 & -0.909 & .801 & \\
\hline PACE68_Amb & 1 & 5 & 3.95 & 0.987 & -0.751 & 0.041 & .820 & \\
\hline \multicolumn{8}{|l|}{ Ambiente } & .824 \\
\hline PACE07_Moti & 1 & 5 & 3.50 & 1.085 & -0.387 & -0.408 & .918 & \\
\hline PACE14_Moti & 1 & 5 & 3.33 & 1.141 & -0.255 & -0.742 & .910 & \\
\hline PACE23_Moti & 1 & 5 & 3.27 & 1.149 & -0.228 & -0.688 & .905 & \\
\hline PACE42_Moti & 1 & 5 & 3.06 & 1.152 & -0.161 & -0.685 & .898 & \\
\hline PACE58_Moti & 1 & 5 & 3.02 & 1.172 & -0.022 & -0.797 & .897 & \\
\hline PACE61_Moti & 1 & 5 & 3.00 & 1.158 & -0.059 & -0.684 & .898 & \\
\hline PACE66_Moti & 1 & 5 & 3.59 & 1.110 & -0.598 & -0.243 & .907 & \\
\hline \multicolumn{8}{|l|}{ Motivación } & .917 \\
\hline PACEO6_Exp & 1 & 5 & 4.03 & 0.967 & -0.792 & 0.168 & .881 & \\
\hline PACE15_Exp & 1 & 5 & 4.04 & 0.949 & -0.777 & 0.232 & .887 & \\
\hline PACE29_Exp & 1 & 5 & 4.18 & 0.839 & -0.800 & 0.253 & .887 & \\
\hline PACE33_Exp & 1 & 5 & 4.19 & 0.894 & -1.043 & 0.853 & .878 & \\
\hline PACE57_Exp & 1 & 5 & 3.68 & 1.037 & -0.475 & -0.384 & .889 & \\
\hline PACE60_Exp & 1 & 5 & 3.82 & 1.068 & -0.645 & -0.270 & .894 & \\
\hline PACE63_Exp & 1 & 5 & 4.02 & 0.953 & -0.979 & 1.020 & .887 & \\
\hline \multicolumn{8}{|l|}{ Expectativas } & .901 \\
\hline PACE08_Rec & 1 & 5 & 3.74 & 1.031 & -0.641 & 0.069 & .888 & \\
\hline PACE13_ReC & 1 & 5 & 3.23 & 1.023 & -0.149 & -0.316 & .878 & \\
\hline PACE21_Rec & 1 & 5 & 3.58 & 1.068 & -0.381 & -0.547 & .878 & \\
\hline PACE32_Rec & 1 & 5 & 3.09 & 1.140 & -0.032 & -0.710 & .868 & \\
\hline PACE34_Rec & 1 & 5 & 3.12 & 1.154 & -0.009 & -0.729 & .873 & \\
\hline PACE41_Rec & 1 & 5 & 3.05 & 1.088 & 0.111 & -0.647 & .868 & \\
\hline PACE44_Rec & 1 & 5 & 3.50 & 1.066 & -0.319 & -0.522 & .882 & \\
\hline PACE50_Rec & 1 & 5 & 3.31 & 1.200 & -0.291 & -0.751 & .893 & \\
\hline \multicolumn{8}{|l|}{ Recursos } & .892 \\
\hline \multicolumn{9}{|c|}{$\begin{array}{l}\text { Nota: } \text { Mín = Mínimo; Máx = Máximo; } M=\text { Media; } D T=\text { Desviación típica; } \text { Seg = Seguridad física; Nor = Normas; Alpro = Relación } \\
\text { alumnado-profesorado; Igu = Relación entre iguales; Coh = Cohesión de grupo; Amb = Aspectos ambientales-estructurales; Moti = } \\
\text { Capacidad de motivación del profesorado; Exp = Percepción expectativas del profesorado; Rec = Recursos metodológicos }\end{array}$} \\
\hline
\end{tabular}


Como puede observarse, la mayoría de los ítems diseñados para evaluar la capacidad de motivación del profesorado y los recursos metodológicos no sólo presentan correlaciones significativas entre los ítems del mismo factor, sino que muestran también correlaciones moderadas y altas con respecto a los ítems del otro factor.

\section{ANÁLISIS FACTORIAL EXPLORATORIO DE LA NUEVA VERSIÓN DE LA ESCALA PACE}

Se verifica que el índice Kaiser-MeyerOlkin (.928) y el de esfericidad de Bartlett $\left(\chi^{2}\right.$ [348] $=15231,55, p<.001)$ cumplen las condiciones necesarias para poder llevar a cabo el AFE (Tabla 5).

Tabla 4

Correlación de cada ítem con el factor propio (discriminación) y con el resto de factores (homogeneidad)

\begin{tabular}{|c|c|c|c|c|c|c|c|c|c|}
\hline Ítems & Seg & Nor & Alpro & Igu & Coh & Amb & Moti & Exp & Rec \\
\hline PACEO1_Seg & $.667^{* \star \star}$ & -.029 & -.029 & .058 & $.120 *$ & .079 & -.047 & -.055 & .009 \\
\hline PACE17_Seg & $.742^{* \star \star}$ & -.097 & -.140 ** & -.024 & .055 & -.026 & $-.138^{\star}$ & -.082 & $-.149 * *$ \\
\hline PACE25_Seg & $.767^{* * *}$ & -.076 & $-.105^{\star}$ & .055 & .101 & .066 & $-.130^{\star}$ & -.090 & -.104 \\
\hline PACE35_Seg & $.661^{* * *}$ & .028 & -.052 & -.014 & .020 & .071 & -.059 & -.076 & -.003 \\
\hline PACE47_Seg & $.505^{\star \star \star}$ & $.154^{\star *}$ & .078 & .035 & .078 & . 077 & .069 & $.147 * \star$ & .017 \\
\hline PACE54_Seg & $.760^{* \star *}$ & -.001 & -.049 & .014 & .072 & .023 & 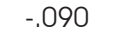 & -.014 & -.107 \\
\hline PACE67_Seg & $.517^{\star \star \star}$ & .061 & .021 & .064 & -.015 & . 084 & -.040 & . 007 & -.070 \\
\hline PACE03_Nor & -.007 & $.692^{\star \star \star}$ & $.282^{\star \star \star}$ & $.203^{* \star *}$ & $.148^{\star \star}$ & $.323^{* \star *}$ & $.260^{* \star *}$ & $.277^{\star * *}$ & $.154^{\star *}$ \\
\hline PACE10_Nor & .012 & $.699 * * *$ & $.305^{\star * \star}$ & $.168^{\star \star}$ & .100 & $.257^{\star \star \star}$ & $.254^{\star \star \star}$ & $.251^{\star \star \star \star}$ & $.151^{\star *}$ \\
\hline PACE20_Nor & -.019 & $.737^{\star \star \star}$ & $.458 * * \star$ & $.205^{\star \star \star}$ & $.223^{\star \star \star}$ & $.456^{\star \star \star}$ & $.412^{\star \star \star}$ & $.385^{\star \star \star}$ & $.327^{\star \star \star}$ \\
\hline PACE30_Nor & -.008 & $.814^{\star \star \star}$ & $.461 * \star *$ & $.169^{\star *}$ & $.163^{\star \star}$ & $.466^{\star \star \star}$ & $.435^{\star \star \star}$ & $.415^{\star \star \star}$ & $.343^{\star \star \star}$ \\
\hline PACE38_Nor & -.010 & $.726^{\star \star \star}$ & $.344^{\star \star *}$ & $.189 * * \star$ & $.194^{\star \star \star}$ & $.398^{\star \star \star}$ & $.344^{\star \star \star}$ & $.327^{\star * \star}$ & $.246^{\star \star \star}$ \\
\hline PACE45_Nor & .092 & $.662^{\star \star \star}$ & $.365^{\star \star \star}$ & .086 & $.145^{\star \star}$ & $.412^{\star \star \star}$ & $.331^{* \star *}$ & $.341^{* \star *}$ & $.248^{\star \star \star}$ \\
\hline PACE52_Nor & .048 & $.638^{* \star *}$ & $.347^{\star \star *}$ & $.160 * \star$ & $.148^{\star \star}$ & $.379 * \star \star *$ & $.270^{\star \star \star}$ & $.374^{\star \star \star}$ & $.206^{\star \star \star}$ \\
\hline PACE64_Nor & -.054 & $.618^{\star \star \star}$ & $.302^{\star \star \star}$ & $.195^{\star \star \star}$ & $.243^{\star \star \star}$ & $.359 * * \star$ & $.326^{* \star *}$ & $.373^{\star * \star}$ & $.233^{\star \star \star}$ \\
\hline
\end{tabular}


Tabla 4 (Continuación)

Correlación de cada ítem con el factor propio (discriminación) y con el resto de factores (homogeneidad)

\begin{tabular}{|c|c|c|c|c|c|c|c|c|c|}
\hline Ítems & Seg & Nor & Alpro & lgu & Coh & Amb & Moti & Exp & Rec \\
\hline PACEO2_Alpro & -.099 & $.408^{\star * \star}$ & $.796^{\star * \star}$ & $.201 * \star \star$ & $.157^{\star \star}$ & $.304^{\star \star \star}$ & $.564^{\star \star \star}$ & $.370 * \star \star$ & $.427^{\star \star *}$ \\
\hline PACE19_Alpro & -.006 & $.457^{\star \star \star}$ & $.787^{\star \star \star}$ & $.216^{\star \star \star}$ & $.144^{\star \star}$ & $.398^{* \star *}$ & $.539 * \star \star$ & $.436^{\star \star *}$ & $.402^{\star \star \star}$ \\
\hline PACE26_Alpro & -.094 & $.337^{* * *}$ & $.820 * \star \star$ & $.299 * * \star$ & $.211 * \star *$ & $.310 * * *$ & $.535^{\star \star \star}$ & $.314^{\star * *}$ & $.406^{* \star *}$ \\
\hline PACE37_Alpro & -.003 & $.403^{\star \star \star}$ & $.784^{\star \star \star}$ & $.296 * \star \star$ & $.219 * \star \star$ & $.369 * \star \star$ & $.557^{\star \star \star}$ & $.365^{\star \star \star}$ & $.433^{\star \star \star}$ \\
\hline PACE46_Alpro & -.039 & $.366^{* \star *}$ & $.833^{\star * \star}$ & $.281^{\star \star \star}$ & $.205^{\star \star *}$ & $.376^{* * *}$ & $.559^{* \star *}$ & $.316^{* * *}$ & $.473^{\star \star *}$ \\
\hline PACE51_Alpro & .012 & $.490 * \star \star$ & $.754^{\star \star \star}$ & $.267 * \star \star$ & $.373^{\star \star \star}$ & $.467 * \star \star$ & $.575^{\star \star \star}$ & $.450 * \star \star$ & $.482^{\star \star \star}$ \\
\hline PACE55_Alpro & -.087 & $.423^{\star \star \star}$ & $.826^{\star \star \star}$ & $.210 * \star \star$ & $.204^{\star \star \star}$ & $.368^{* \star *}$ & $.581^{\star \star \star}$ & $.432^{\star \star \star}$ & $.462^{\star \star \star}$ \\
\hline PACE65_Alpro & -.027 & $.405^{\star \star \star}$ & $.777^{\star * \star}$ & $.199 * * \star$ & $.258 * \star *$ & $.378^{* \star *}$ & $.592^{\star \star *}$ & $.432^{\star * \star}$ & $.487 * \star \star$ \\
\hline PACE05_Igu & .024 & $.317^{\star \star \star}$ & $.305^{\star \star \star}$ & $.780 * \star \star$ & $.473^{\star \star *}$ & $.171^{\star \star}$ & $.192^{\star \star \star}$ & $.251^{\star \star \star}$ & $.117^{\star}$ \\
\hline PACE1 1_lgu & .043 & $.153^{\star \star}$ & $.249 * * *$ & $.850 * \star \star$ & $.397 * \star *$ & $.117^{\star}$ & $.144^{\star \star}$ & .090 & .088 \\
\hline PACE16_lgu & .062 & $.218 * * \star$ & $.296 * \star \star$ & $.847^{\star \star \star}$ & $.486^{\star \star \star}$ & $.185^{\star \star}$ & $.180 * \star$ & $.192 * \star \star$ & $.122^{\star}$ \\
\hline PACE24_lgu & .085 & $.213^{\star \star \star}$ & $.218^{\star \star \star}$ & $.772^{\star \star \star}$ & $.593^{\star * \star}$ & $.200 * \star \star$ & $.203^{\star \star \star}$ & $.265^{\star \star \star}$ & $.194^{\star \star \star}$ \\
\hline PACE28_Igu & -.012 & $.205^{\star \star \star}$ & $.275^{\star \star \star}$ & $.835^{\star * \star}$ & $.583^{\star * *}$ & $.230 * * *$ & $.220 * \star *$ & $.221 * * *$ & $.165^{\star *}$ \\
\hline PACE40_lgu & .027 & $.135^{\star}$ & $.211^{\star \star \star}$ & $.846^{\star \star \star}$ & $.551^{\star \star \star}$ & $.126^{*}$ & $.142^{\star \star}$ & $.134^{*}$ & .086 \\
\hline PACE49_Igu & .015 & $.180 * \star$ & $.217^{\star * \star}$ & $.776^{\star \star \star}$ & $.517 * \star \star$ & $.200 * * \star$ & $.144^{\star *}$ & $.171^{* *}$ & $.126^{\star}$ \\
\hline PACEO4_Coh & .037 & $.211^{* \star *}$ & $.184^{\star \star}$ & $.603 * * \star$ & $.706^{\star \star \star}$ & $.313^{* \star *}$ & $.214^{\star \star \star}$ & $.270 * \star \star$ & $.261^{* \star *}$ \\
\hline PACE12_Coh & $.190 * * *$ & $.244^{\star \star \star}$ & $.141^{* \star}$ & $.366 * \star \star$ & $.658^{\star \star \star}$ & $.368^{\star \star \star}$ & $.213^{\star \star \star}$ & $.286^{\star \star \star}$ & $.169 * *$ \\
\hline PACE18_Coh & .100 & $.139 *$ & $.175^{\star \star}$ & $.446^{\star \star \star}$ & $.794^{\star * \star}$ & $.297 * \star \star$ & $.267^{* \star *}$ & $.195^{\star \star *}$ & $.217 * * *$ \\
\hline PACE27_Coh & .043 & $.190 * * *$ & $.330 * * *$ & $.511^{\star \star \star}$ & $.822^{\star \star \star}$ & $.336^{* \star *}$ & $.345^{\star * *}$ & $.335^{\star * *}$ & $.244^{\star \star *}$ \\
\hline
\end{tabular}


Tabla 4 (Continuación)

Correlación de cada ítem con el factor propio (discriminación) y con el resto de factores (homogeneidad)

\begin{tabular}{|c|c|c|c|c|c|c|c|c|c|}
\hline Ítems & Seg & Nor & Alpro & Igu & Coh & Amb & Moti & Exp & Rec \\
\hline PACE36_Coh & .076 & $.191^{\star * \star}$ & $.195^{\star \star \star}$ & $.519 * \star *$ & $.805^{\star \star *}$ & $.276^{\star * \star}$ & $.254^{\star * \star}$ & $.295^{\star * *}$ & $.173^{\star *}$ \\
\hline PACE43_Coh & .056 & .087 & $.221^{\star \star \star}$ & $.442^{\star \star \star}$ & $.789 * \star \star$ & $.300 * \star \star$ & $.338 * \star \star$ & $.305^{\star \star \star}$ & $.264^{\star \star \star}$ \\
\hline PACE56_Coh & .010 & $.252^{\star * *}$ & $.253^{\star \star *}$ & $.515^{\star \star \star}$ & $.782^{\star \star \star}$ & $.323^{* * *}$ & $.275^{\star \star \star}$ & $.380 * * *$ & $.203^{* \star *}$ \\
\hline PACE62_Coh & .036 & $.163^{\star \star}$ & $.180 * \star$ & 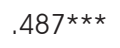 & $.800 * \star \star$ & $.318^{\star \star *}$ & $.262^{\star * *}$ & $.315^{\star \star \star}$ & $.206^{\star * \star}$ \\
\hline PACEO9_Amb & .040 & $.419 * * *$ & $.400 * \star *$ & $.148^{\star \star}$ & $.301 * * \star$ & $.733^{\star * *}$ & $.481 * * *$ & $.315^{\star * *}$ & $.466^{* \star *}$ \\
\hline PACE22_Amb & .100 & $.300 * * *$ & $.283^{* * *}$ & $.145^{\star \star}$ & $.421^{\star \star *}$ & $.655^{\star \star \star}$ & $.389 * * *$ & $.328^{* * *}$ & $.447^{\star * \star}$ \\
\hline PACE31_Amb & .052 & $.478^{\star \star \star}$ & $.376 * * \star$ & $.145^{\star \star}$ & $.281^{\star \star \star}$ & $.716^{\star \star \star}$ & $.454^{\star \star \star}$ & $.381 * \star *$ & $.414^{\star \star \star}$ \\
\hline PACE39_Amb & .005 & $.369 * * *$ & $.252^{\star \star \star}$ & $.136^{\star \star}$ & $.246^{\star \star \star}$ & $.690 * \star \star$ & $.264^{\star \star \star}$ & $.289 * \star \star$ & $.324^{\star \star *}$ \\
\hline PACE48_Amb & .005 & $.369 * * \star$ & $.252^{\star \star \star}$ & $.136^{*}$ & $.246^{\star \star \star}$ & $.690^{* * *}$ & $.264^{\star * *}$ & $.289^{\star \star *}$ & $.324^{\star \star \star}$ \\
\hline PACE53_Amb & .083 & $.329 * \star \star$ & $.241^{\star \star \star}$ & .082 & $.204^{\star \star \star}$ & $.657^{* * \star}$ & $.282^{\star * *}$ & $.298^{\star * \star}$ & $.325^{\star \star \star}$ \\
\hline PACE59_Amb & .082 & $.307 * \star \star *$ & $.367^{\star \star \star}$ & .066 & 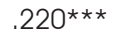 & $.705^{\star \star \star}$ & $.361^{* \star *}$ & $.275^{\star \star \star}$ & $.391 * \star \star$ \\
\hline PACE68_Amb & .006 & $.320 * * *$ & $.246^{* \star *}$ & $.254^{\star \star \star}$ & $.301^{\star \star \star}$ & $.551^{\star \star \star}$ & $.254^{\star \star \star}$ & $.343^{\star \star *}$ & $.254^{\star \star \star}$ \\
\hline PACEO7_Moti & -.032 & $.461^{* \star *}$ & $.520 * \star \star$ & $.207 * \star \star$ & $.355^{\star \star \star}$ & $.476 * \star \star$ & $.707 * \star \star$ & $.502^{\star \star \star}$ & $.527^{\star \star \star}$ \\
\hline PACE14_Moti & $-.140 * *$ & $.372 * \star \star$ & $.524^{\star \star \star}$ & $.177^{\star \star}$ & $.257 * \star \star$ & $.394^{* * *}$ & $.782^{\star \star \star}$ & $.448^{* * *}$ & $.559 * \star *$ \\
\hline PACE23_Moti & -.102 & $.369 * * \star$ & $.635^{\star * \star}$ & $.141^{\star \star}$ & $.255^{\star \star \star}$ & $.354^{\star \star *}$ & $.819 * \star \star$ & $.520 * \star \star$ & $.628^{* * *}$ \\
\hline PACE42_Moti & -.117 & $.330 * * *$ & $.619 * \star \star$ & $.173^{\star \star}$ & $.307^{* * *}$ & $.435^{\star \star \star}$ & $.866^{\star \star \star}$ & $.543^{\star \star *}$ & $.655^{\star \star \star}$ \\
\hline PACE58_Moti & -.013 & $.393^{\star * \star}$ & $.596 * \star \star$ & $.176^{\star \star}$ & $.303^{* \star *}$ & $.449 * * *$ & $.874^{\star * *}$ & $.500 * \star *$ & $.668^{* \star \star}$ \\
\hline PACE61_Moti & -.035 & $.342^{* * *}$ & $.585^{\star \star *}$ & $.175^{\star \star}$ & $.302^{* * *}$ & $.434^{* * *}$ & $.866^{\star \star \star}$ & $.478^{\star \star \star}$ & $.659 * \star \star$ \\
\hline PACE66_Moti & -.100 & $.425^{\star * \star}$ & $.543^{* \star *}$ & $.169 * \star$ & $.245^{\star \star \star}$ & $.435^{\star \star \star}$ & $.804^{\star * *}$ & $.578 * \star \star$ & $.598^{\star \star *}$ \\
\hline PACEO6_Exp & -.043 & $.362 * \star \star$ & $.347 * \star \star$ & $.211 * \star \star$ & $.323^{\star \star \star}$ & $.367 \star \star \star$ & $.507 \star \star \star$ & $.825^{\star \star \star}$ & $.458^{\star \star \star}$ \\
\hline
\end{tabular}


Tabla 4 (Continuación)

Correlación de cada ítem con el factor propio (discriminación) y con el resto de factores (homogeneidad)

\begin{tabular}{|c|c|c|c|c|c|c|c|c|c|}
\hline Ítems & Seg & Nor & Alpro & Igu & Coh & Amb & Moti & Exp & Rec \\
\hline PACE15_Exp & .015 & $.419^{* * *}$ & $.295^{* * *}$ & $.207^{\star \star \star}$ & $.407^{\star \star \star}$ & $.337^{\star \star \star}$ & $.353^{* \star *}$ & $.785^{\star \star \star}$ & $.360^{* * *}$ \\
\hline PACE29_Exp & -.001 & $.400^{\star \star \star \star}$ & $.340^{\star \star \star}$ & $.183^{\star \star}$ & $.338^{\star \star \star}$ & $.360^{\star \star \star}$ & $.419^{* * *}$ & $.780^{\star \star \star}$ & $.369^{\star \star *}$ \\
\hline PACE33_EXP & .021 & $.383^{\star \star \star}$ & $.340^{\star \star \star}$ & $.189 \star \star \star$ & $.344^{\star \star \star}$ & $.346^{\star \star \star}$ & $.433^{\star \star \star}$ & $.844^{\star \star \star}$ & $.394^{\star \star \star}$ \\
\hline PACE57_Exp & -.082 & $.380^{\star \star \star}$ & $.468^{\star \star \star}$ & $.127^{\star}$ & $.251^{\star \star \star}$ & $.387^{\star \star \star \star}$ & $.611^{* * *}$ & $.785^{\star \star \star}$ & $.602^{\star \star \star}$ \\
\hline PACE60_EXP & -.079 & $.410^{\star \star \star}$ & $.471^{\star \star \star}$ & $.196^{\star \star \star}$ & $.254^{\star \star \star}$ & $.430^{\star \star \star}$ & $.628^{\star \star \star}$ & $.759 * \star \star$ & $.614^{\star \star \star}$ \\
\hline PACE63_Exp & -.010 & $.348^{\star \star \star}$ & $.395^{\star \star \star}$ & $.151^{\star *}$ & $.250 * \star \star$ & $.371^{\star \star \star}$ & $.472^{\star \star \star}$ & $.786^{\star \star \star *}$ & $.475^{\star \star \star}$ \\
\hline PACE08_Rec & -.047 & $.171^{\star \star}$ & $.394^{\star \star \star}$ & .087 & $.183^{\star *}$ & $.361^{\star \star \star}$ & $.458^{\star \star \star}$ & $.423 * \star \star *$ & $.663^{* * *}$ \\
\hline PACE13_Rec & -.026 & $.211^{\star \star \star}$ & $.406^{\star \star \star}$ & $.145^{\star \star}$ & $.239 * \star \star$ & $.399 * \star \star$ & $.560^{\star \star *}$ & $.480^{\star \star \star}$ & $.756^{\star \star \star}$ \\
\hline PACE21_Rec & -.044 & $.293^{\star * \star}$ & $.434^{\star \star \star}$ & $.142^{\star \star}$ & $.256^{\star \star \star}$ & $.477^{\star \star \star}$ & $.596^{\star * \star}$ & $.475^{\star \star \star}$ & $.762^{\star \star *}$ \\
\hline PACE32_Rec & $-.150 * \star$ & $.286^{\star \star \star}$ & $.436^{\star \star \star}$ & $.123^{\star}$ & $.239 * \star \star$ & $.455^{\star \star \star}$ & $.655^{\star \star \star}$ & .476 & $.837^{\star \star *}$ \\
\hline PACE34 Rec & - .102 & $.244^{\star \star \star}$ & 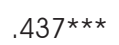 & $.118^{*}$ & 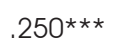 & $.465^{\star \star \star}$ & $.615^{\star \star \star}$ & $.470^{\star \star \star *}$ & $.800^{\star \star \star}$ \\
\hline PACE41_Rec & - 102 & $.307^{\star \star \star}$ & $.526^{\star \star \star}$ & $.135^{\star}$ & $.241^{\star \star \star}$ & $.467^{\star \star \star *}$ & $.678^{* * *}$ & 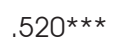 & $.838^{* * *}$ \\
\hline PACE44_Rec & -.053 & $.260^{\star \star \star}$ & $.395^{\star \star \star}$ & .078 & $.155^{\star \star}$ & $.337^{\star \star \star}$ & $.506^{\star \star *}$ & $.439 * \star *$ & $.730^{* * *}$ \\
\hline PACE50_Rec & -.007 & $.269^{\star * \star}$ & $.349^{\star \star \star}$ & . $110^{*}$ & $.147^{\star *}$ & $.378^{\star \star \star}$ & $.471^{\star \star \star}$ & $.337 * \star \star$ & $.661^{* * *}$ \\
\hline \multicolumn{10}{|c|}{$\begin{array}{l}\text { Nota: } \text { Seg }=\text { Seguridad física; } \text { Nor }=\text { Normas; Alpro }=\text { Relación alumnado-profesorado; lgu }=\text { Relación entre iguales; } \text { Coh }=\text { Cohesión de } \\
\text { grupo; } A m b=\text { Aspectos ambientales-estructurales; Moti }=\text { Capacidad de motivación del profesorado; Exp }=\text { Percepción expectativas del } \\
\text { profesorado; Rec }=\text { Recursos metodológicos. }{ }^{*} p<.05 ;{ }^{* *} p<.01 ;{ }^{* * *} p<.001\end{array}$} \\
\hline
\end{tabular}

Tabla 5

Comunalidades y pesos factoriales de los ítems de la escala PACE

\begin{tabular}{|c|c|c|c|c|c|c|c|c|c|c|}
\hline \multirow{2}{*}{ Ítems } & \multicolumn{10}{|c|}{ Factores } \\
\cline { 2 - 11 } & COm & 1 & 2 & 3 & 4 & 5 & 6 & 7 & 8 & 9 \\
\hline $\begin{array}{c}\text { PACE01_ } \\
\text { Seg }\end{array}$ & .378 & .027 & .021 & .596 & .095 & .069 & -.056 & -.072 & .003 & .042 \\
\hline $\begin{array}{c}\text { PACE17_ } \\
\text { Seg }\end{array}$ & .598 & .076 & -.029 & .759 & .105 & -.031 & .000 & -.040 & .059 & -.100 \\
\hline $\begin{array}{c}\text { PACE25_ } \\
\text { Seg }\end{array}$ & .634 & .091 & .065 & .771 & .112 & .058 & -.113 & -.021 & .076 & -.056 \\
\hline $\begin{array}{c}\text { PACE35_ } \\
\text { Seg }\end{array}$ & .379 & -.033 & -.016 & .601 & -.037 & .110 & .017 & .016 & .035 & .131 \\
\hline $\begin{array}{c}\text { PACE47_ } \\
\text { Seg }\end{array}$ & .183 & -.034 & -.046 & .356 & -.145 & -.145 & .107 & -.027 & -.034 & -.011 \\
\hline
\end{tabular}


Tabla 5 (Continuación)

Comunalidades y pesos factoriales de los ítems de la escala PACE

\begin{tabular}{|c|c|c|c|c|c|c|c|c|c|c|}
\hline \multirow{2}{*}{ Ítems } & \multicolumn{10}{|c|}{ Factores } \\
\hline & Com & 1 & 2 & 3 & 4 & 5 & 6 & 7 & 8 & 9 \\
\hline PACE54_Seg & .564 & -.026 & -.038 & .741 & -.049 & -.021 & .139 & -.060 & -.007 & -.013 \\
\hline PACE67_Seg & .134 & -.073 & .081 & .308 & .001 & -.060 & -.095 & .124 & -.055 & -.059 \\
\hline PACEO3_Nor & .444 & -.021 & .089 & -.022 & -.667 & -.012 & -.009 & .044 & .027 & -.030 \\
\hline PACE10_Nor & .404 & .012 & .087 & .002 & -.633 & .001 & .034 & .101 & -.038 & -.044 \\
\hline PACE20_Nor & .427 & .034 & .007 & -.025 & -.589 & -.007 & -.083 & -.037 & -.135 & .003 \\
\hline PACE30_Nor & .625 & .076 & .002 & .001 & -.707 & -.018 & -.049 & .051 & -.087 & .020 \\
\hline PACE38_Nor & .509 & .041 & .028 & -.043 & -.600 & -.015 & -.059 & -.028 & -.017 & -.008 \\
\hline PACE45_Nor & .383 & .005 & -.098 & .063 & -.483 & -.076 & -.112 & -.022 & -.123 & -.040 \\
\hline PACE52_Nor & .352 & -.046 & .047 & .033 & -.446 & -.134 & -.124 & .078 & -.078 & -.016 \\
\hline PACE64_Nor & .346 & .067 & .026 & -.091 & -.472 & -.132 & -.077 & -.083 & .022 & -.055 \\
\hline PACEO2_Alpro & .588 & .074 & .131 & -.049 & .029 & .065 & -.015 & .005 & -.761 & -.015 \\
\hline PACE19_Alpro & .658 & .091 & .035 & .029 & -.025 & -.140 & -.169 & .113 & -.714 & -.144 \\
\hline PACE26_Alpro & .631 & .109 & .038 & -.064 & -.053 & -.062 & -.008 & .053 & -.687 & -.048 \\
\hline PACE37_Alpro & .579 & .037 & .108 & .008 & -.011 & .117 & -.036 & .019 & -.749 & .090 \\
\hline PACE46_Alpro & .658 & .037 & .118 & .030 & -.044 & .014 & -.039 & .024 & -.672 & .047 \\
\hline PACE51_Alpro & .632 & -.133 & -.046 & .023 & -.221 & .001 & .041 & -.229 & -.570 & .179 \\
\hline PACE55_Alpro & .627 & .023 & .001 & -.054 & -.079 & -.047 & .006 & -.011 & -.706 & .042 \\
\hline PACE65_Alpro & .609 & -.058 & -.081 & .012 & -.103 & -.030 & .102 & -.145 & -.663 & .160 \\
\hline PACE05_lgu & .593 & -.040 & .665 & .008 & -.203 & -.046 & .102 & -.057 & -.084 & -.009 \\
\hline PACE11_Igu & .712 & .076 & .859 & .044 & .000 & .092 & -.003 & .082 & -.083 & -.007 \\
\hline PACE16_Igu & .681 & .010 & .785 & .048 & -.025 & -.011 & -.025 & -.001 & -.110 & -.027 \\
\hline PACE24_lgu & .589 & -.071 & .609 & .046 & -.091 & -.065 & .083 & -.224 & .059 & .151 \\
\hline PACE28_lgu & .674 & .034 & .715 & -.050 & .020 & -.028 & -.071 & -.160 & -.052 & -.020 \\
\hline PACE40_lgu & .689 & .084 & .770 & -.007 & .045 & .023 & -.036 & -.131 & -.011 & -.061 \\
\hline PACE49_Igu & .561 & -.143 & .645 & -.025 & -.046 & .041 & -.003 & -.156 & -.023 & .126 \\
\hline
\end{tabular}


Tabla 5 (Continuación)

Comunalidades y pesos factoriales de los ítems de la escala PACE

\begin{tabular}{|c|c|c|c|c|c|c|c|c|c|c|}
\hline \multirow{2}{*}{ Ítems } & \multicolumn{10}{|c|}{ Factores } \\
\hline & Com & 1 & 2 & 3 & 4 & 5 & 6 & 7 & 8 & 9 \\
\hline PACEO4_Coh & .571 & -.159 & .431 & -.033 & -.073 & -.011 & -.048 & -.376 & .119 & .256 \\
\hline PACE12_Coh & .403 & -.019 & .106 & .150 & -.121 & -.093 & -.131 & -.451 & .108 & .010 \\
\hline PACE18_Coh & .621 & .047 & .102 & .066 & -.025 & .080 & .038 & -.740 & .009 & .041 \\
\hline PACE27_Coh & .668 & .035 & .140 & .001 & .091 & -.081 & -.020 & -.699 & -.199 & -.085 \\
\hline PACE36_Coh & .612 & -.001 & .196 & .015 & -.016 & -.114 & .014 & -.658 & .000 & -.062 \\
\hline PACE43_Coh & .631 & .173 & .114 & .015 & .180 & -.078 & -.060 & -.689 & -.051 & -.044 \\
\hline PACE56_Coh & .603 & -.021 & .183 & -.059 & .023 & -.233 & -.100 & -.584 & -.070 & -.143 \\
\hline PACE62_Coh & .580 & .018 & .180 & -.016 & .018 & -.107 & -.060 & -.635 & .047 & -.014 \\
\hline PACEO9_Amb & .502 & .109 & -.031 & .062 & -.083 & -.055 & -.650 & -.018 & .032 & .033 \\
\hline PACE22_Amb & .444 & -.042 & -.155 & .066 & .009 & .013 & -.623 & -.124 & -.246 & .084 \\
\hline PACE31_Amb & .465 & .035 & -.098 & .050 & -.353 & .068 & -.264 & -.174 & -.031 & .218 \\
\hline PACE39_Amb & .582 & -.046 & .017 & -.062 & -.067 & -.031 & -.727 & .001 & .010 & .027 \\
\hline PACE48_Amb & .530 & -.053 & .110 & .019 & -.198 & -.002 & -.592 & .054 & .014 & .098 \\
\hline PACE53_Amb & .362 & .120 & -.086 & .051 & -.262 & .187 & -.320 & -.197 & -.069 & .245 \\
\hline PACE59_Amb & .427 & -.146 & .079 & -.127 & .067 & -.207 & -.392 & -.017 & .308 & -.146 \\
\hline PACE68_Amb & .265 & -.066 & .085 & -.053 & -.064 & -.158 & -.325 & -.111 & -.048 & .025 \\
\hline PACEO7_Moti & .471 & .221 & -.020 & -.027 & -.171 & -.157 & -.130 & -.156 & -.195 & .101 \\
\hline PACE14_Moti & .522 & .408 & .024 & -.099 & -.133 & -.067 & -.044 & -.065 & -.181 & .154 \\
\hline PACE23_Moti & .642 & .343 & -.057 & -.028 & -.088 & -.114 & .121 & -.098 & -.348 & .243 \\
\hline PACE42_Moti & .725 & .730 & -.042 & .083 & -.043 & -.061 & .021 & -.086 & .168 & .022 \\
\hline PACE58_Moti & .750 & .745 & -.016 & -.033 & .131 & .047 & -.056 & -.079 & .082 & .113 \\
\hline PACE61_Moti & .755 & .863 & -.003 & -.055 & .032 & .014 & -.003 & -.071 & .063 & .040 \\
\hline PACE66_Moti & .603 & .420 & .018 & -.050 & -.159 & -.208 & -.035 & -.003 & -.139 & .181 \\
\hline PACEO6_Exp & .695 & .190 & .075 & -.020 & .041 & -.740 & -.145 & .032 & .039 & .040 \\
\hline PACE15_Exp & .690 & -.122 & -.051 & -.003 & -.132 & -.729 & .005 & -.184 & .009 & .033 \\
\hline
\end{tabular}


Tabla 5 (Continuación)

Comunalidades y pesos factoriales de los ítems de la escala PACE

\begin{tabular}{|c|c|c|c|c|c|c|c|c|c|c|}
\hline \multirow{2}{*}{ Ítems } & \multicolumn{10}{|c|}{ Factores } \\
\hline & Com & 1 & 2 & 3 & 4 & 5 & 6 & 7 & 8 & 9 \\
\hline PACE29_Exp & .609 & .027 & -.054 & -.004 & -.095 & -.687 & -.053 & -.126 & -.021 & -.020 \\
\hline PACE33_Exp & .741 & -.033 & -.036 & .033 & -.063 & -.497 & .030 & -.091 & -.013 & .075 \\
\hline PACE57_Exp & .596 & .189 & .018 & -.041 & -.060 & -.467 & -.014 & .061 & -.045 & .322 \\
\hline PACE60_Exp & .577 & .206 & .081 & -.026 & -.130 & -.364 & .002 & .060 & -.023 & .367 \\
\hline PACE63_Exp & .538 & -.011 & .007 & .017 & -.037 & -.579 & -.011 & .040 & -.074 & .243 \\
\hline PACE08_Rec & .415 & -.102 & -.027 & -.017 & .137 & -.169 & -.054 & -.001 & -.209 & .510 \\
\hline PACE13_ReC & .536 & .116 & .080 & .016 & .092 & -.129 & -.048 & .026 & -.049 & .607 \\
\hline PACE21_Rec & .531 & .081 & .027 & -.004 & -.014 & -.078 & -.126 & -.009 & -.067 & .565 \\
\hline PACE32_Rec & .695 & .217 & .057 & -.103 & -.040 & -.022 & -.066 & .015 & .040 & .683 \\
\hline PACE34_Rec & .608 & .133 & .008 & -.076 & .062 & -.064 & -.115 & -.038 & -.058 & .794 \\
\hline PACE41_Rec & .679 & .112 & .004 & -.069 & .016 & -.089 & -.044 & -.010 & -.149 & .634 \\
\hline PACE44_ReC & .430 & .031 & .022 & -.010 & .028 & -.154 & -.055 & .099 & -.107 & .527 \\
\hline PACE50_Rec & .358 & .149 & .034 & .076 & -.106 & .031 & -.070 & .042 & -.005 & .470 \\
\hline Autoval & & 18.471 & 6.308 & 3.601 & 3.283 & 2.897 & 2.292 & 1.742 & 1.503 & 1.176 \\
\hline$\%$ Varianza e & licada & 27.163 & 9.277 & 5.295 & 4.828 & 4.261 & 3.371 & 2.562 & 2.211 & 1.729 \\
\hline
\end{tabular}

Se extrae una solución compuesta por nueve factores con un porcentaje de varianza total explicada del $60.70 \%$ y unos valores superiores a .30 para las comunalidades en la gran mayoría de los ítems (Tabla 5). Los ítems que presentan las comunalidades más bajas son PACE47 (.183), PACE67 (.134) y PACE68 (.265).

En cuanto a los pesos factoriales de los ítems, la gran mayoría de ellos obtienen saturaciones por encima del .40 exigible, y muchos de ellos se acercan, e incluso superan, el valor más restrictivo de .70 . No obstante, nueve ítems no cumplen con el criterio establecido (PACE07, PACE23, PACE31,
PACE47, PACE53, PACE59, PACE60, PACE67 e PACE68). Asimismo, todos los ítems cuyos pesos factoriales superan el mínimo exigido saturan en el factor para el que fueron diseñados, a excepción del PACE04; este ítem alcanza una mayor saturación en el factor relación entre iguales que en el de cohesión, en contra de la previsión inicial.

\section{SELECCIÓN DE LOS ÍTEMS CON MEJORES PROPIEDADES PSICOMÉTRICAS}

Tras analizar las propiedades psicométricas de los diferentes ítems, junto con los aspectos conceptuales que motivaron la creación de 
cada uno de ellos, se procede a desechar los ítems con peores características, dejando en cada factor un mínimo de tres. En los factores seguridad, normas, relación alumnadoprofesorado, relación entre iguales, cohesión de grupo y aspectos ambientales-estructurales se opta por seleccionar cuatro ítems en lugar de 3 , dado que se considera necesario para asegurar la representatividad teórica de esas dimensiones. Así, se seleccionan 33 de los 68 ítems que conformaban en un principio la nueva versión de la escala PACE (Anexo 1). A la versión de 33 ítems obtenida se le nombra PACE-33.

\section{CONSISTENCIA INTERNA Y VARIANZA MEDIA EXTRACTADA}

En la Tabla 6 se muestran los diferentes coeficientes de fiabilidad de consistencia interna de las subescalas del PACE-33. Además, también se presenta la VME como indicador de la validez de su estructura interna.

Respecto a la fiabilidad de la consistencia interna, todas las subescalas muestran índices adecuados. Estos índices están comprendidos entre .753 y .927 para el alfa de Cronbach; entre .743 y .863 para el omega de McDonald; y entre .748 y .864 para la rho de Raykov. Los valores más altos en el alfa de Cronbach se observan en las subescalas capacidad de motivación del profesorado $(\alpha=.927)$ y relación entre iguales $(\alpha=.890)$; y en los coeficientes de fiabilidad compuesta, en las subescalas relación entre iguales $(\omega=.863$, $\rho=.864)$, relación alumnado-profesorado $(\omega$ $=.822, \rho=.823)$ y capacidad de motivación del profesorado $(\omega=.822, \rho=.824)$.

Respecto a la varianza media extractada, la mayoría de las subescalas presentan valores adecuados, esto es, valores en torno o superiores a .50. Los factores normas (VME $=.426)$ y aspectos ambientales-estructurales (VME =.422), sin embargo, manifiestan valores inferiores.

\section{VALIDEZ CONVERGENTE}

Tal y como puede comprobarse en la Tabla 7, las nueve subescalas de la medida PACE33 muestran correlaciones estadísticamente significativas y positivas con las dos subescalas del cuestionario CECSCE. El grado de asociación entre las distintas subescalas varía entre baja y alta. Concretamente, las correlaciones encontradas entre las subescalas del PACE-33 y el clima referente al centro oscilan entre .255 y .627 ; con respecto al clima referente al profesorado, se hallan correlaciones entre .133 y .731.

Tabla 6

Coeficientes de consistencia interna y Varianza Media Extraída

\begin{tabular}{|c|c|c|c|c|}
\hline & $\begin{array}{c}\text { Alfa de } \\
\text { Cronbach }(\alpha)\end{array}$ & $\begin{array}{c}\text { Omega de } \\
\text { McDonald }(\omega)\end{array}$ & $\begin{array}{c}\text { rho de Raykov } \\
(\rho)\end{array}$ & VME \\
\hline Seguridad física & .805 & .809 & .811 & .520 \\
\hline Normas & .797 & .747 & .748 & .537 \\
\hline Relación alumnado- profesorado & .852 & .822 & .864 & .615 \\
\hline Relación entre iguales & .890 & .863 & .791 & .486 \\
\hline Cohesión grupo & .868 & .790 & .744 & .422 \\
\hline Aspectos ambientales-estructurales & .753 & .743 & .762 & .611 \\
\hline $\begin{array}{c}\text { Capacidad motivación del } \\
\text { profesorado }\end{array}$ & .927 & .822 & .748 & .517 \\
\hline Expectativas profesorado & .847 & .762 & & .500 \\
\hline Recursos metodológicos & .874 & .745 & & .748 \\
\hline Nota: VME= Varianza Media Extraída
\end{tabular}


Tabla 7

Coeficientes de correlación de Pearson entre las subescalas del PACE-33 y las del CECSCE

\begin{tabular}{|c|c|c|c|c|}
\hline & \multicolumn{2}{|c|}{ Clima centro } & \multicolumn{2}{|c|}{ Clima profesorado } \\
\hline & $r$ & $p$ & $r$ & $p$ \\
\hline Seguridad física & .255 & $.001 * *$ & .174 & $.028^{*}$ \\
\hline Normas & .388 & $<.001^{\star \star \star}$ & .439 & $<.001^{\star \star \star}$ \\
\hline Relación alumnado- profesorado & .487 & $<.001^{\star \star \star}$ & .689 & $<.001^{\star \star *}$ \\
\hline Relación entre iguales & .271 & $.001 * \star$ & .133 & $.042^{\star}$ \\
\hline Cohesión grupo & .521 & $<.001^{* *}$ & .210 & $.008 * \star$ \\
\hline Aspectos ambientales-estructurales & .570 & $<.001 * * *$ & .449 & $<.001 * * \star$ \\
\hline $\begin{array}{l}\text { Capacidad motivación del } \\
\text { profesorado }\end{array}$ & .627 & $<.001^{\star \star \star}$ & .751 & $<.001^{* \star *}$ \\
\hline Expectativas profesorado & .370 & $<.001^{\star \star}$ & .436 & $<.001 * \star \star$ \\
\hline Recursos metodológicos & .589 & $<.001^{* * *}$ & .534 & $<.001^{\star \star}$ \\
\hline
\end{tabular}

La capacidad de motivación del profesorado es de todas las subescalas del PACE-33 la que se relaciona en mayor grado con las dos relativas al CECSCE. Por el contrario, las subescalas seguridad física y relación entre iguales del PACE-33 son las que presentan menor grado de asociación con el clima referente al centro y el clima referente al profesorado, respectivamente.

\section{DISCUSIÓN}

Son abundantes los estudios que han abordado el clima escolar desde el siglo XX. Sin embargo, hoy en día aún no se conoce con exactitud la estructura interna de este constructo y no se dispone de un instrumento de medida validado en el contexto español que posibilite la evaluación de los aspectos más relevantes del clima escolar de manera fiable (Escalante, Fernández-Zabala et al., 2020; Escalante, Goñi et al., 2020). Por ello, los dos objetivos de este estudio son rediseñar la escala PACE y presentar evidencias de su fiabilidad y validez. Además, es esperable que el estudio de la dimensionalidad de esta escala pueda ayudar a clarificar la estructura interna del clima escolar.
Respecto al rediseño del PACE, se obtiene una nueva versión compuesta por 68 ítems, cuyas propiedades psicométricas han sido analizadas para completar el segundo objetivo de este estudio.

El análisis factorial exploratorio realizado respalda la estructura dimensional de nueve factores obtenida en el estudio previo realizado (Escalante, Goñi et al., 2020): seguridad, normas, relación alumnado-profesorado, relación entre iguales, cohesión de grupo, aspectos ambientales-estructurales, capacidad de motivación del profesorado, expectativas del profesorado y recursos metodológicos. Estos nueve aspectos específicos han sido evaluados previamente por separado por autores como López-González y Bisquerra (2013), López-González et al. (2012), Bear et al. (2011) o Guo et al. (2011).

La valoración conjunta de las propiedades psicométricas de los diferentes ítems junto con el análisis de contenido de los mismos, permite la selección de los ítems con mejores garantías técnicas (sustantivas y métricas). Con el fin de lograr un cuestionario parsimonioso, en este estudio se seleccionan tres ítems por factor, el mínimo aconsejado en investigaciones previas (Carpenter, 2018; 
Ferrando y Anguiano-Carrasco, 2010), incluyendo un cuarto ítem en aquellos factores en los que resulta necesario para asegurar su representatividad conceptual. El instrumento de medida resultante consta de 33 ítems (PACE-33).

Merece un comentario especial el hecho de que numerosos ítems de los factores capacidad de motivación del profesorado y recursos metodológicos muestren correlaciones moderadas y altas tanto entre los ítems del mismo factor como con respecto a los ítems del otro factor. No es de extrañar que los ítems de estos factores compartan un rasgo en común ya que los ítems del factor recursos metodológicos hacen referencia al uso de recursos motivacionales por parte del profesorado, siendo este tipo de recursos, precisamente, una de las estrategias más eficaces de las que dispone el profesorado para motivar al alumnado (Blázquez, Orcos, Mainz, y Sáez, 2018). Futuras investigaciones deberán ahondar a ese respecto para clarificar si se trata de factores que presentan una alta correlación entre sí o si, por el contrario, estas asociaciones podrían ser un indicativo de la existencia de un factor de orden superior que los englobase.

La escala PACE-33 presenta resultados adecuados de fiabilidad. Se comprueba que todas las subescalas presentan adecuados coeficientes de consistencia interna (Hair et al., 2018). Sin embargo, la varianza media extractada presenta resultados más modestos en las subescalas normas y aspectos ambientales-estructurales, aunque valorando los datos obtenidos junto con los de la fiabilidad compuesta y las correlaciones ítem-total corregidas (REFS), los valores son aceptables (Bettencourt, 2004). Por tanto, estos valores de fiabilidad por tratarse de medidas de consistencia interna, garantizan la eficacia de la escala PACE-33 a la hora de interpretar los datos obtenidos como representación adecuada del clima escolar autopercibido. Dicho de otro modo, en este instrumento de medida las puntuaciones obtenidas en cada una de las subescalas reflejan con precisión los aspectos específicos más importantes de este constructo en la adolescencia. Asimismo, esta escala obtiene resultados positivos de validez externa.

Además de presentar propiedades psicométricas adecuadas, hay que subrayar que, a diferencia de la gran mayoría de las medidas disponibles hasta el momento para evaluar la percepción del clima escolar, el PACE-33 es una escala breve que contiene únicamente 33 ítems para la medida de nueve factores. Este hecho cobra una especial relevancia en el ámbito educativo, donde es necesario contar con instrumentos breves ante posibles limitaciones de tiempo en su aplicación, la fatiga del alumnado participante o la aplicación de un gran número de instrumentos simultáneamente (Esnaola, Freeman, Sarasa, Fernández-Zabala, y Axpe, 2016).

Se concluye, por tanto, que la escala PACE33 es un instrumento válido, fiable y breve que permite conocer los aspectos más relevantes que explicarían la percepción que tiene el alumnado de ESO y Bachillerato acerca del clima escolar. El uso de esta escala permitiría, además, conocer en mayor profundidad cómo perciben los/las adolescentes el clima de su propio centro escolar e identificar aquellos aspectos susceptibles de ser mejorados para favorecer un clima escolar positivo. Esto, a su vez, posibilitaría el diseño de intervenciones educativas ajustadas a las necesidades específicas de cada centro. Una medida completa, rica, del clima escolar de un centro en concreto ayudaría en un diagnóstico eficaz, posibilitando intervenciones específicas de mejora o de refuerzo de aquellos aspectos que más aporten a la convivencia escolar.

En cualquier caso, este estudio no está exento de limitaciones. Como limitación principal hay que señalar que, si bien se ha contado con una muestra heterogénea y adecuada para los análisis llevados a cabo, esta no es muy amplia y ha sido seleccionada de manera incidental. Además, hay que subrayar el carácter exploratorio de este estudio. Futuras investigaciones deberán corroborar los resultados obtenidos en este trabajo mediante el empleo de procedimientos como el análisis factorial confirmatorio en muestras más amplias y aleatorias. Esta perspectiva de trabajo permitirá corroborar la fiabilidad 
y validez de la escala y, a su vez, someter a prueba la dimensionalidad del modelo teórico que subyace al PACE-33.

- Conflicto de intereses.

Los autores declaran no tener ningún conflicto de intereses.

\section{REFERENCIAS}

Aldridge, J. M., y Ala'i, K. (2013). Assessing students' views of school climate: Developing and validating the What's Happening In This School? (WHITS) questionnaire. Improving Schools, 16(1), 47-66.

Aron, A. M., Milicic, N., y Armijo, I. (2012). Clima social escolar: una escala de evaluación -Escala de Clima Social Escolar, ECLIS-. Universitas Psychologica, $17(3)$, 803-813.

Bandalos, D. L., y Finney, S. J. (2010). Factor analysis: Exploratory and confirmatory. En G. R. Hancock y R. O. Mueller (Eds.). The reviewer's guide to quantitative methods in the social sciences (pp. 93-114). Nueva York, NY: Routledge.

Bear, G. G., Gaskins, C., Blank, J., y Chen, F. F. (2011). Delaware School Climate SurveyStudent: Its factor structure, concurrent validity, and reliability. Journal of School Psychology, 49(2), 157-174. https://doi. org/10.1016/i.jsp.2011.01.001

Bentler, P. M. (2005). EQS 6 Structural equations program manual. Encino, Los Ángeles, LA: Multivariate Software.

Bettencourt, L. (2004). Change-oriented organizational citizenship behaviors: The direct and moderating influence of goal orientation. Journal of Retailing, 80(3), 165-180. https://doi.org/10.1016/i. jretai.2003.12.001

Bisquerra, R. (2004). Metodología de la investigación educativa. Madrid, España: La Muralla.

Blázquez, P. J., Orcos, L., Mainz, J., y Saez, D. (2018). Propuesta metodológica para la mejora del aprendizaje de los alumnos a través de la utilización de las impresoras 3D como recurso educativo en el aprendizaje basado en proyectos. Psicología, Conocimiento y Sociedad 8(1), 162-193. https://doi.org/10.26864/PCS. v8.n 1.8
Carretero-Dios, H., y Pérez, C. (2005). Normas para el desarrollo y revisión de estudios instrumentales. International Journal of Clinical and Health Psychology, $5(3), 521-551$.

Carpenter, S. (2018). Ten steps in scale development and reporting: A guide for researchers. Communication Methods and Measures, 12(1), 25-44.

Cemalcilar, Z. (2010). Schools as socialisation contexts: Understanding the impact of school climate factors on students sense of school belonging. Applied Psychology: An International Review, 59(2), 243272. http://dx.doi.org/10.1111/i.14640597.2009.00389.x.

Cho, E. (2016). Making reliability reliable: A systematic approach to reliability coefficients. Organizational Research Methods, 19(4), 651-682. https://doi. org/10.1177/1094428116656239

Cohen, J. (2006). Social, emotional, ethical, and academic education: Creating a climate for learning, participation in democracy, and well-being. Harvard Educational Review, 76(2), 201 $237 . \quad$ http://dx.doi.org/10.17763/ haer.76.2.j44854x1524644vn

Cohen, J., McCabe, E. M., Michelli, N. M., y Pickeral, T. (2009). School climate: Research, policy, teacher education and practice. Teachers College Record, $117(1)$, 180-213.

Daily, S. M., Mann, M. J., Kristjansson, A. L., Smith, M. L., y Zullig, K. J. (2019). School climate and academic achievement in middle and high school students. Journal of School Health, 89(3), 173-180. https:// doi.org/10.1111/josh.12726

Escalante, N., Fernández-Zabala, A., Goñi, E., e Izar-de-la-Fuente, I. (2020). Eskola giroa konstruktuaren berrikuspen teorikoa. Tantak, 32(1), 117-136.

Escalante, N., Goñi, E., Fernández-Zabala, A., e Izar-de-la-Fuente, I. (2020). Diseño y estructura factorial del cuestionario Percepción del Alumnado sobre el Clima Escolar (PACE). European Journal of Education and Psychology, 13(1), 287-302. https://doi.org/10.30552/ejep.v13i1.294

Esnaola, I., Freeman, J., Sarasa, M., Fernández-Zabala, A., y Axpe, I. (2016). 
Validity evidence based on internal structure of scores of the Emotional QuotientInventory: Youth Version Short (EQ-i: YV-S) in a Spanish sample. The Spanish Journal of Psychology, 19(e12), 1-19.

Farina, K. A. (2019). Promoting a culture of bullying: Understanding the role of school climate and school sector. Journal of School Choice, 13(1), 94-120. https://doi.org/10 $.1080 / 15582159.2018 .1526615$

Ferrando, P. J., y Anguiano-Carrasco, C. (2010). El análisis factorial como técnica de investigación en psicología. Papeles del Psicológo, 31 (1), 18-33.

Flora, D. B., LaBrish, C., y Chalmers, R. P. (2012). Old and new ideas for data screening and assumption testing for exploratory and confirmatory factor analysis. Frontiers in Quantitative Psychology and Measurement, 3(55), 1-21.

Furlong, M. J. y Morrison, R. L. (1995). Status update of research related to National Education Goal Seven: School violence content area. En Proceedings of National Education Goals Panel/National Alliance of Pupil Service Organization Safe School, Safe Students Conference. Educational Research Information Centre, Chapel Hill, NC.

Gravetter, F., y Wallnau, L. (2014). Essentials of statistics for the behavioral sciences $\left(8^{a}\right.$ ed.). Belmont, Massachusetts: Wadsworth.

Guo, P., Choe, J., y Higgins-D'Alessandro, A. (2011). Report of construct validity and internal consistency findings for the comprehensive school climate inventory. Nueva York, NY: Fordham University.

Hair, J. F., Anderson, R. E., Tatham, R. L., y Black, W. C. (2004). Análisis Multivariante. Madrid, España: Pearson.

Hair, J., Babin, B. Anderson, R., y Black, W. (2018). Multivariate data analysis (8nd ed.). Hampshire, Winchester: Cengage Learning.

Haynes, N. M., Emmons, C. L., y Comer, J. P. (1993). Elementary and middle school climate survey. New Haven, CT: Yale University Child Study Center.

Herrera, K., y Rico, R. (2014). El clima escolar como elemento fundamental de la convivencia en la escuela. Escenarios, 12(2), 7-18. http://dx.doi.org/10.15665/ esc.v12i2.311
Hong, J. S., Espelage, D. L., y Lee, J. M. (2018). School climate and bullying prevention programs. En $H$. Shapiro (Ed.), The Wiley handbook on violence in education (pp. 359-374). Hoboken, NJ: John Wiley \& Sons. https://doi. org/10.1002/9781118966709.ch17

Jackson, D. (1970). A sequential system for personality scale development. En C. D. Spielberger (Ed.), Current topics in clinical and community psychology (Vol. 2, pp. 6196). Nueva York, NY: Academic Press.

Kaiser, H. (1970). A second generation little jiffy. Psychometrika, 35(4), $401-415$. http:// dx.doi.org/10.1007/BF02291817

Lloret-Segura, S., Ferreres-Traver, A., Hernández-Baeza, A., y Tomás-Marco, I. (2014). El análisis factorial exploratorio de los ítems: una guía práctica, revisada y actualizada. Anales de Psicología, 30(3), 1-18. https://doi.org/10.6018/ analesps.30.3.199361

López-González, L., y Bisquerra, R. (2013). Validación y análisis de una escala breve para evaluar el clima de clase en Educación Secundaria. ISEP Science, 5, 62- 77.

López-González, L., y Oriol, X. (2016). La relación entre competencia emocional, clima de aula y rendimiento académico en estudiantes de secundaria. Cultura y Educación, 28(1), 130-156. https://doi.org /10.1080/11356405.2015.1120448

López-González, E., Tourón, J., y Tejedor, F. J. (2012). Diseño de un micro instrumento para medir el clima de aprendizaje en cuestionarios de contexto. Bordón, 64(2), $111-126$.

Maxwell, S., Reynolds, K. J., Lee, E., Subasic, E., y Bromhead, D. (2017). The impact of school climate and school identification on academic achievement: Multilevel modeling with student and teacher data. Frontiers in Psychology, 8(1), 1-21. https:// doi.org/10.3389/fpsyg.2017.02069

Montero, I., y León, O. G. (2007). A guide for naming research studies in psychology. International Journal of Clinical and Health Psychology, 7(3), 847-862.

Montoya, O. (2007). Aplicación del análisis factorial a la investigación de mercados. Caso de estudio. Scientia et Technica, 35, $281-286$. 
Morales, P. (2011). El análisis factorial en la construcción e interpretación de tests, escalas y cuestionarios. Madrid, España: Universidad Pontificia Comillas.

Muñiz, J. (2018). Introducción a la Psicometría. Teoría clásica y TRI. Madrid, España: Pirámide.

National School Climate Center. (2014). School climate guidelines. Recuperado de https://www.schoolclimate.org/about/ourapproach/guidelines

Newland, L. A., DeCino, D. A., Mourlam, D. J., y Strous, G. A. (2019). School climate, emotions, and relationships: Children's experiences of well-being in the Midwestern U.S. International Journal of Emotional Education Special Issue, $17(1), 67-83$.

Nunnally, J., y Bernstein, I. (1995). Teoría psicométrica. Ciudad de México, México: McGraw-Hill.

Pérez, A., Ramos, G., y López, E. (2010). Clima social aula: percepción diferenciada de los alumnos de Educación Secundaria Obligatoria. Cultura y Educación, 22(3), 259-281. http://dx.doi. org/10.1174/113564010804932184

Quijada, A., Ruiz, M. A., Huertas, J. A., y Alonso-Tapia, J. (2020). Development and validation of the School Climate Questionnaire for Secondary and High School Teachers (SCQ-SHST). Anales de Psicología, 36(1), 155-165. https://doi. org/10.6018/analesps.341001

Rosenblatt, J., y Furlong, M. J. (1997). Assessing the reliability and validity of student selfreports of campus violence. Journal of Youth and Adolescence, 26(2), 187-201. https:// doi.org/10.1023/A:1024552531672
Sun, L., y Royal, K. (2017). School climate in American secondary schools: A psychometric examination of PISA 2009 school climate scale. Journal of Curriculum and Teaching, 6(2), 6-12. https://doi. org/10.5430/ict.v6n2p6

Thapa, A., Cohen, J., Guffey, S., y HigginsD'Alessandro, A. (2013). A review of school climate research. Review of Educational Research, 83(3), 357-385. http://dx.doi. $\mathrm{org} / 10.3102 / 0034654313483907$

Trianes, M. V., Blanca, M. J., de la Morena, L., Infante, L., y Raya, S. (2006). Un cuestionario para evaluar el clima social del centro escolar. Psicothema, 18(2), 272-277.

Turner, I., Reynolds, K. J., Lee, E., Subasic, E., y Bromhead, D. (2014). Well-being, school climate, and the social identity process: A latent growth model study of bullying perpetration and peer victimization. School Psychology Quarterly 29(3), 320-335. https://doi.org/10.1037/spq0000074

Varela, J. J., Sirlopú, D., Melipillán, R., Espelage, D., Green, J., y Guzmán, J. (2019). Exploring the influence school climate on the relationship between school violence and adolescent subjective wellbeing. Child Indicators Research, 12(6), 2095-2110. https://doi.org/10.1007/ s12187-019-09631-9

Yang, C., Bear, G. G., Chen, F. F., Zhang, W., Blank, J. C., y Huang, X. (2013). Students' perceptions of school climate in the U.S. and China. School Psychology Quarterly, 28(1), 7-24. doi: http://dx.doi. org/10.1037/spq00000027 
ANEXO 1

NUEVA VERSIÓN DE LA ESCALA PERCEPCIÓN DEL ALUMNADO SOBRE EL CLIMA ESCOLAR

\begin{tabular}{|c|c|c|c|c|}
\hline $\begin{array}{c}\text { Totalmente } \\
\text { desacuerdo }\end{array}$ & En Desacuerdo & $\begin{array}{c}\text { Ni de acuerdo. ni } \\
\text { desacuerdo }\end{array}$ & De acuerdo & $\begin{array}{c}\text { Totalmente de } \\
\text { acuerdo }\end{array}$ \\
\hline
\end{tabular}

\begin{tabular}{|c|c|}
\hline & Ítems \\
\hline 1. & En este centro hay alumnos/as que destruyen cosas. \\
\hline 2. & En este centro cuento con profesores/as a los que puedo contar los problemas que me surgen. \\
\hline 3. & En este centro tenemos establecidas normas claras. \\
\hline 4. & Los/las alumnos/as tenemos buena comunicación entre nosotros/as. \\
\hline 5. & En este centro tengo compañeros/as en los que puedo confiar. \\
\hline 6. & Los/las profesores/as esperan que los/las alumnos/as tengamos interés por aprender. \\
\hline 7. & Los/as estudiantes somos valorados públicamente cuando realizamos un buen trabajo. \\
\hline 8. & $\begin{array}{l}\text { Los/las profesores/as, además del libro de texto de cada asignatura, utilizan otros recursos (materiales creados por } \\
\text { ellos/as mismos/as, esquemas, proyectos, etc.). }\end{array}$ \\
\hline 9. & Mi centro está bien conservado. \\
\hline 10. & Las normas se nos explican claramente a los/las alumnos/as. \\
\hline 11. & Me siento a gusto hablando con mis compañeros/as de mis problemas. \\
\hline 12. & $\begin{array}{l}\text { Los/las alumnos/as nos respetamos mutuamente independientemente de nuestras características individuales } \\
\text { (sexo, etnia, forma de pensar, ritmo de aprendizaje, etc.). }\end{array}$ \\
\hline 13. & Los recursos que utilizan los/las profesores/as son innovadores. \\
\hline 14. & Los/las profesores/as felicitan a los/las alumnos/as que ayudan a otros/as alumnos/as. \\
\hline 15. & Los/las profesores/as esperan que trabajemos duro. \\
\hline 16. & En este centro cuento con compañeros/as a los que puedo contar los problemas que me surgen. \\
\hline 17. & En este centro hay alumnos/as que se meten en peleas (empujones, patadas, etc.). \\
\hline 18. & En esta clase hay un buen ambiente y una buena convivencia. \\
\hline 19. & Los/las profesores/as están disponibles cuando necesito hablar con ellos/as. \\
\hline 20. & Las normas están visibles para todos/as. \\
\hline 21. & Los/las profesores/as proponen actividades para trabajar en grupo. \\
\hline 22. & Mi clase se mantiene limpia y ordenada. \\
\hline 23. & Los/las profesores/as nos motivan. \\
\hline 24. & Es fácil hablar con mis compañeros/as. \\
\hline 25. & En este centro hay alumnos/as que amenazan o insultan a otros/as alumnos/as. \\
\hline 26. & Me siento cómodo/a hablando con los/las profesores/as de los problemas que me surgen. \\
\hline 27. & En esta clase los/las alumnos/as nos cuidamos y ayudamos mutuamente. \\
\hline 28. & Mis compañeros/as están disponibles cuando necesito hablar con ellos/as. \\
\hline 29. & Los/las profesores/as esperan que los/las alumnos/as demos lo mejor de nosotros/as mismos/as. \\
\hline 30. & En este centro está muy claro lo que está permitido y lo que está prohibido. \\
\hline 31. & Los demás espacios del centro se mantienen limpios y bien cuidados. \\
\hline
\end{tabular}




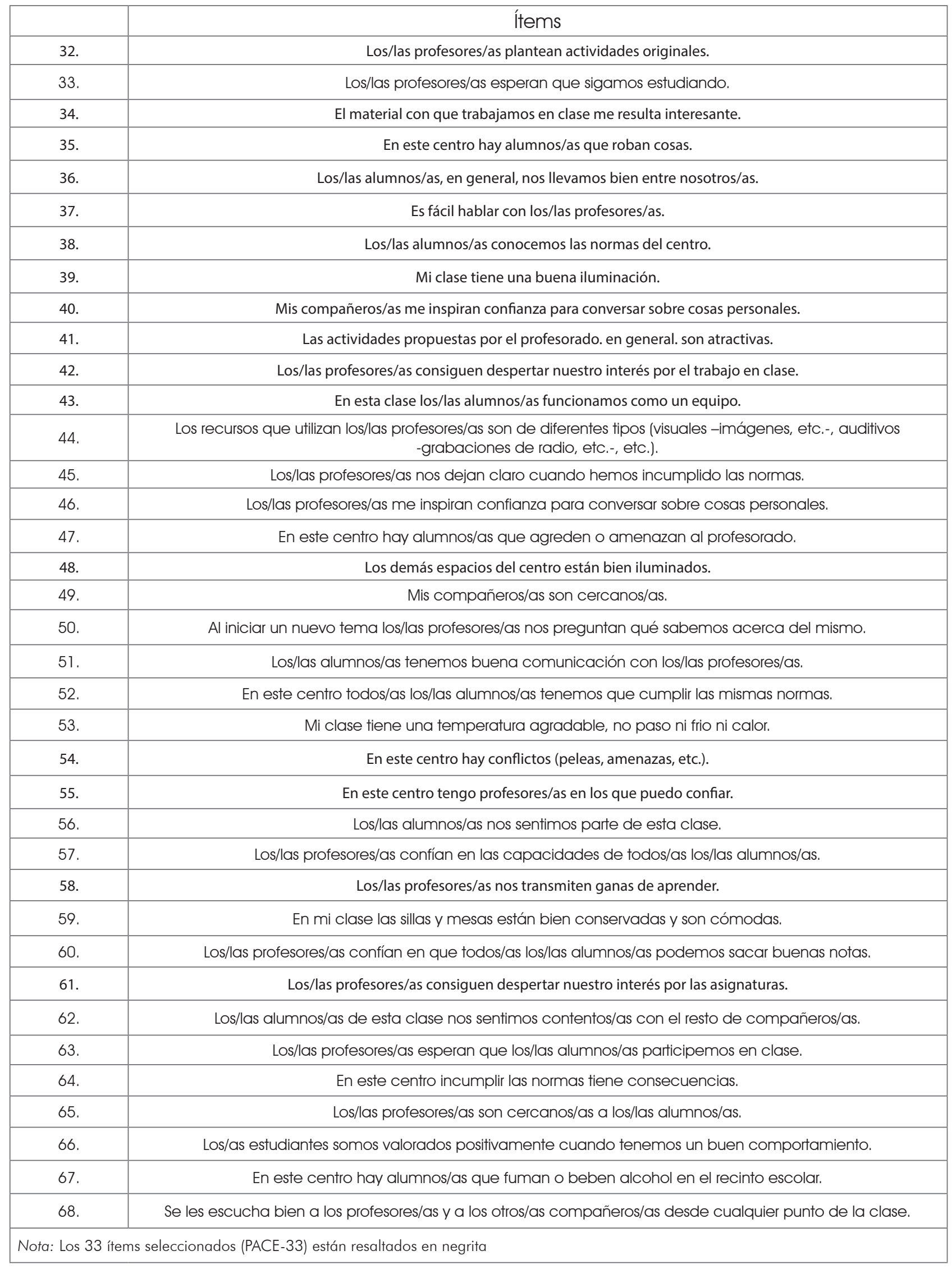

